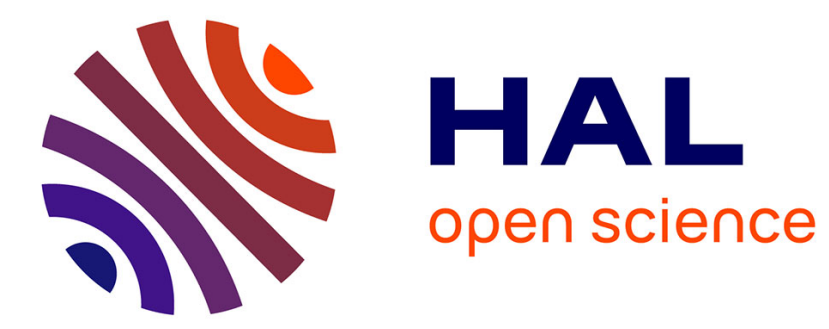

\title{
Lifting in Sobolev spaces
}

Jean Bourgain, Haïm Brezis, Petru Mironescu

\section{To cite this version:}

Jean Bourgain, Haïm Brezis, Petru Mironescu. Lifting in Sobolev spaces. Journal d'analyse mathématique, 2000, 80, pp.37-86. 10.1007/BF02791533 . hal-00747691

\section{HAL Id: hal-00747691 \\ https://hal.science/hal-00747691}

Submitted on 1 Nov 2012

HAL is a multi-disciplinary open access archive for the deposit and dissemination of scientific research documents, whether they are published or not. The documents may come from teaching and research institutions in France or abroad, or from public or private research centers.
L'archive ouverte pluridisciplinaire HAL, est destinée au dépôt et à la diffusion de documents scientifiques de niveau recherche, publiés ou non, émanant des établissements d'enseignement et de recherche français ou étrangers, des laboratoires publics ou privés. 


\title{
LIFTING IN SOBOLEV SPACES
}

\author{
Jean Bourgain $^{(1)}$, Haim Brezis ${ }^{(2),(3)}$ and Petru Mironescu(4)
}

\section{Introduction.}

Let $\Omega \subset \mathbb{R}^{n}$ be a (smooth) bounded domain which is connected and simply connected. Given a function $u: \Omega \rightarrow S^{1}$ (i.e., $u: \Omega \rightarrow \mathbb{C}$ and $|u(x)|=1$ a.e.) we may write pointwise

$$
u(x)=e^{i \varphi(x)}
$$

for some function $\varphi: \Omega \rightarrow \mathbb{R}$. The objective is to find a lifting $\varphi$ "as regular as $u$ permits." For example, if $u$ is continuous one may choose $\varphi$ to be continuous and if $u \in C^{k}$ one may also choose $\varphi$ to be $C^{k}$. A more delicate result asserts that if $u \in \mathrm{VMO}$ (= vanishing means oscillation), then one may choose $\varphi$ to be also VMO (see R. Coifman and Y. Meyer [1] and H. Brezis and L. Nirenberg [1]). In this paper we study the question of lifting in the framework of the Sobolev spaces $W^{s, p}$ with $0<s<\infty$ and $1<p<\infty$. The motivation comes from problems of the Ginzburg-Landau type where one considers questions such as Min $\int|\nabla u|^{2}$ in the class of functions $u: \Omega \rightarrow S^{1}$ (see e.g. F. Bethuel, H. Brezis and F. Hélein [1]).

The first result in that direction is

Theorem (F. Bethuel and X. Zheng [1]). Assume

$$
u \in W^{1, p}\left(\Omega ; S^{1}\right) \quad \text { with } p \geq 2
$$

then $u$ may be written as $u=e^{i \varphi}$ for some $\varphi \in W^{1, p}(\Omega ; \mathbb{R})$.

Surprisingly the restriction $p \geq 2$ is optimal in any dimension $n \geq 2$, i.e., given any $p<2$ there is some $u \in W^{1, p}$ which cannot be lifted by a $\varphi \in W^{1, p}$ (such examples will be given later; see Section 4).

We address the same questions in all Sobolev spaces $W^{s, p}$. Here is a summary of our main results:

Theorem 1. Assume $n=1,0<s<\infty$ and $1<p<\infty$. Then the answer to the lifting question in $W^{s, p}$ is always positive. 
Theorem 2. Assume $n \geq 2,0<s<1$ and $1<p<\infty$. The answer to the lifting question in $W^{s, p}$ is:

a) positive if $s p<1$,

b) negative if $1 \leq s p<n$,

c) positive if $s p \geq n$.

Theorem 3. Assume $n \geq 2,1 \leq s<\infty$ and $1<p<\infty$. The answer to the lifting question in $W^{s, p}$ is:

a) negative if $s p<2$,

b) positive if $s p \geq 2$.

In these statements "positive" means that every $u \in W^{s, p}\left(\Omega ; S^{1}\right)$ may be written as $u=e^{i \varphi}$ for some $\varphi \in W^{s, p}(\Omega ; \mathbb{R})$ and "negative" means that for some $u$ 's in $W^{s, p}\left(\Omega ; S^{1}\right)$ there is no $\varphi \in W^{s, p}(\Omega ; \mathbb{R})$ such that $u=e^{i \varphi}$.

As a simple consequence of the theorems when $p=2$, i.e., for $H^{s}=W^{s, 2}$, we have

Corollary 1. When $n=1$ the answer to the lifting problem in $H^{s}$ is always positive.

When $n \geq 2$ the answer to the lifting problem in $H^{s}$ is:

a) positive if $0<s<1 / 2$,

b) negative if $1 / 2 \leq s<1$,

c) positive if $s \geq 1$.

The proof of Theorems 1 and 2 when $s p<1$ turns out to be quite involved (even for the $H^{s}$ case, $s<1 / 2$, and even when $n=1$ ). It relies on a characterization, due to G. Bourdaud [1] (see also the earlier paper of R. Devore and V. A. Popov [1]), of the $W^{s, p}$ space when $s p<1$; for the convenience of the reader, and also because we make use of sharp estimates, we have presented a proof in a separate section, Appendix A.

In view of the Corollary for $n \geq 2$, a function $u \in H^{1 / 2}\left(\Omega ; S^{1}\right)$ need not have a lifting $\varphi \in H^{1 / 2}(\Omega ; \mathbb{R})$; however, it has a lifting $\varphi$ in $H^{s}, \forall s<1 / 2$. We prove (see Appendix E)

Theorem 4. Assume $Q$ is a cube in $\mathbb{R}^{n}, n \geq 1$. For every $u \in H^{s}\left(Q ; S^{1}\right)$ with $0<s<1 / 2$ one may find a $\varphi$ in $H^{s}$ such that $u=e^{i \varphi}$ and satisfying the (optimal) estimate

$$
\|\varphi\|_{H^{s}} \leq C(1-2 s)^{-1 / 2}\|u\|_{H^{s}}
$$

with $C$ independent of $u$ and independent of $s$ (for $s$ near 1/2).

Such an estimate is useful in deriving bounds for the Ginzburg-Landau functional when the boundary condition belongs to $H^{1 / 2}$. For example, let $Q$ be a cube of $\mathbb{R}^{n}, n \geq 1$, and let $\Omega=Q \times(0,1)$. For any function $g \in H^{1 / 2}(Q ; \mathbb{C})$, set

$$
\begin{aligned}
& H_{g}^{1}\left(\Omega=\left\{u(x, t): \Omega \rightarrow \mathbb{C} ; \int_{\Omega}|\nabla u|^{2} d x d t<\infty \text { and } u(x, 0)=g(x) \text { on } Q\right\},\right. \\
& E_{\varepsilon}(u)=\frac{1}{2} \int_{\Omega}|\nabla u|^{2}+\frac{1}{4 \varepsilon^{2}} \int_{\Omega}\left(|u|^{2}-1\right)^{2},
\end{aligned}
$$


where $\nabla$ denotes the full gradient (in $(x, t))$.

Theorem 5. For every $g \in H^{1 / 2}\left(Q ; S^{1}\right)$ we have, for $\varepsilon>0$,

$$
E_{\varepsilon}=\operatorname{Min}_{u \in H_{g}^{1}(\Omega)} E_{\varepsilon}(u) \leq C \log (1 / \varepsilon)\|g\|_{H^{1 / 2}}^{2}
$$

where $C$ is independent of $\varepsilon$ and of $g$.

For variants of Theorem 5, see Remark 8 in Section 5.

The plan of the paper is the following:

1. Proof of Theorems 1 and 2 when $s p<1$

2. Proof of Theorem 1 when $s p \geq 1$ and of Theorem 2 when $s p \geq n$

3. Proof of Theorem 3 when $s p \geq 2$

4. Examples of obstruction in Theorems 2 and 3

5. Control of lifting in the $H^{s}$-norm for $s \overrightarrow{<} \frac{1}{2}$ and application to Ginzburg-Landau

Appendix A. A characterization of $W^{s, p}(\Omega ; \mathbb{R})$ when $s p<1$

Appendix B. Functions in $W^{s, p}(\Omega ; \mathbb{Z})$ are constant when $s p \geq 1$

Appendix C. Composition in fractional Sobolev spaces

Appendix D. Gagliardo-Nirenberg inequalities and products in fractional Sobolev spaces

Appendix E. Behaviour of the $H^{s}$-norms of lifting for $s \overrightarrow{<} \frac{1}{2}$. Proof of Theorem 4

Appendix F. Martingale representation and lifting in $H^{s, p}$

\section{Proof of Theorems 1 and 2 when $\mathrm{sp}<1$.}

Here, the assumption that $\Omega$ is simply connected is not needed since we may always extend the given function by a constant outside $\Omega$; the resulting function still belongs to $W^{s, p}$ since $s p<1$ (this is a well-known fact, see e.g. Lions-Magenes [1], Section 1.11 when $p=2$ and the references therein; it is also a consequence of the characterization of $W^{s, p}$ in Appendix A). Thus, we may assume that $\Omega=(0,1)^{n}$ and we use the same notation as in Appendix A.

Let $u \in W^{s, p}\left(\Omega ; S^{1}\right)$. For each $j=0,1, \ldots$, consider the function $U_{j} \in \mathcal{E}_{j}$ defined by

$$
U_{j}(x)= \begin{cases}\frac{E_{j}(u)(x)}{\left|E_{j}(u)(x)\right|} & \text { if } E_{j}(u)(x) \neq 0 \\ 1 & \text { if } E_{j}(u)(x)=0\end{cases}
$$

Clearly $U_{j} \rightarrow u$ a.e. on $\Omega$ (since $E_{j}(u) \rightarrow u$ a.e. and $|u|=1$ a.e.) For each $j=0,1, \ldots$ we construct a real-valued function $\varphi_{j} \in \mathcal{E}_{j}$ such that

$$
e^{i \varphi_{j}}=U_{j} \quad \text { on } \Omega
$$




$$
\left|\varphi_{j}-\varphi_{j-1}\right| \leq C\left|U_{j}-U_{j-1}\right| \quad \text { on } \Omega \text {. }
$$

Note that (1.2) can be achieved by induction on $j$, for example with $C=\pi / 2$.

On the other hand, observe that for every $\xi, \eta, \zeta \in \mathbb{C}$ with $|\zeta|=1$, we have

$$
\left|\frac{\xi}{|\xi|}-\frac{\eta}{|\eta|}\right| \leq 4(|\zeta-\xi|+|\zeta-\eta|)
$$

with the convention that $\frac{0}{0}=1$ (consider separately the case where $|\xi|,|\eta| \geq 1 / 2$ and the case where either $|\xi|<1 / 2$ or $|\eta|<1 / 2)$.

Applying (1.3) to $\xi=E_{j}(u)(x), \eta=E_{j-1}(u)(x)$ and $\zeta=u(x)$ we obtain a.e. on $\Omega$

$$
\left|U_{j}-U_{j-1}\right| \leq 4\left(\left|u-E_{j}(u)\right|+\left|u-E_{j-1}(u)\right|\right) .
$$

Combining this with (1.2) yields

$$
\left|\varphi_{j}-\varphi_{j-1}\right| \leq C\left(\left|u-E_{j}(u)\right|+\left|u-E_{j-1}(u)\right|\right)
$$

and thus

$$
\sum_{j \geq 1} 2^{s p j}\left\|\varphi_{j}-\varphi_{j-1}\right\|_{L^{p}}^{p} \leq C \sum_{j \geq 0} 2^{s p j}\left\|u-E_{j}(u)\right\|_{L^{p}}^{p} .
$$

Applying Theorem A.1 and Corollary A.1 in Appendix A, we conclude that $\varphi_{j} \rightarrow \varphi$ in $L^{p}$ with $\varphi \in W^{s, p}, e^{i \varphi}=u$, and

$$
\|\varphi\|_{W^{s, p}} \leq C\|u\|_{W^{s, p}} .
$$

We may always assume (by adding to $\varphi$ an integer multiple of $2 \pi$ ) that

$$
\left|\int_{\Omega} \varphi\right| \leq 2 \pi \text {. }
$$

Thus, we have constructed a function $\varphi \in W^{s, p}$ such that $e^{i \varphi}=u$ and

$$
\|\varphi\|_{L^{p}}+\|\varphi\|_{W^{s, p}} \leq C\left(1+\|u\|_{W^{s, p}}\right) .
$$

Remark 1. One should observe the linear dependence while in the continuous case there is no bound whatsoever for $\|\varphi\|_{L^{\infty}}$ in terms of $\|u\|_{L^{\infty}}$; see also Remark 3 where we show that there is no bound for $\varphi$ in $H^{1 / 2}$ in terms for $\|u\|_{H^{1 / 2}}$ in one dimension despite the fact that every $u \in H^{1 / 2}$ has a (unique) lifting in $H^{1 / 2}$.

Remark 2. The function $\varphi$ constructed above also belongs to every $L^{q}, q<\infty$. This may be easily seen by observing that $u \in W^{s, p} \cap L^{\infty} \subset W^{\sigma, q}$ for every $\sigma<s$ with $\sigma q=s p$ (by the Gagliardo-Nirenberg inequality, see Appendix D). Therefore $\varphi$ belongs to every such $W^{\sigma, q}$. Choosing $\sigma$ close to zero we obtain a $q$ which is arbitrarily large. 


\section{Proof of Theorem 1 when $s p \geq 1$ and of Theorem 2 when $s p \geq n$.}

When $s p>1$ in Theorem 1 or $s p>n$ in Theorem 2, $u$ is continuous by the Sobolev imbedding theorem and, locally, we may consider $\varphi=-i \log u$ which is well-defined and singlevalued. To conclude, we rely on a lemma about composition:

Lemma 1. Assume $n \geq 1,0<s<\infty$ and $1<p<\infty$. Let $v \in W^{s, p}(\Omega) \cap L^{\infty}(\Omega)$ and let $\Phi \in C^{\infty}$. Then $\Phi \circ v \in W^{s, p}(\Omega)$.

The proof is very simple when $0<s<1$ (using the definition of $W^{s, p}$ and the fact that $\Phi$ is Lipschitz on the range of $v$ ). This lemma is also well-known when $s$ is an integer, with the help of the Gagliardo-Nirenberg inequality. When $s>1$ is not an integer the argument is more delicate; we refer to Escobedo [1] and Lemma C.1 in Appendix C.

We now turn to the proof of Theorem 1 when $s=1 / p$; the proof of Theorem 2 when $s=n / p$ is identical and we omit it. Set $I=\Omega=(0,1)$.

By standard trace theory there is some $\tilde{u} \in W^{s+1 / p, p}\left(I^{2} ; \mathbb{R}^{2}\right)$ such that

$$
\tilde{u}(x, 0)=u(x) .
$$

Since $u$ takes its values into $S^{1}$ one may expect that, near $I \times\{0\}, \tilde{u}$ takes its values "close" to $S^{1}$. This is not true for a general extension $\tilde{u}$. However, special extensions have that property. For example

$$
\tilde{u}(x, y)=\frac{1}{2 y} \int_{x-y}^{x+y} u(t) d t
$$

( $u$ is extended by symmetry to the interval $(-2,+2)$ ) has the property that $\tilde{u} \in W^{s+1 / p, p}$, and moreover, $|\tilde{u}(x, y)| \rightarrow 1$ uniformly in $x$ as $y \rightarrow 0$. This is a consequence of the fact that $W^{s, p} \subset \mathrm{VMO}$ in the limiting case of the Sobolev imbedding (see e.g. Boutet de Monvel-Berthier, Georgescu and Purice [1],[2], Brezis and Nirenberg [1]). Similarly, any harmonic extension $\tilde{u}$ of $u$ in $I^{2}$ has also the same property (see Brezis and Nirenberg [2], Appendix 3). If we consider $v=\tilde{u} /|\tilde{u}|$ in a neighborhood $\omega$ of $I \times\{0\}$ in $I^{2}$ we have an extension $v$ of $u$ such that

$$
v \in W^{s+1 / p, p}\left(\omega ; S^{1}\right) .
$$

Here, we have used again Lemma 1.

Let us now explain how to complete the proof of the theorem when $p=2$, i.e., $u \in H^{1 / 2}\left(I ; S^{1}\right)$. From the above discussion we have some extension $v$ of $u$, with

$$
v \in H^{1}\left(\omega ; S^{1}\right) .
$$

Applying the theorem of Bethuel and Zheng we may write

$$
v=e^{i \psi}
$$

for some $\psi \in H^{1}(\omega ; \mathbb{R})$ and then $\varphi=\psi_{\left.\right|_{I}}$ has the required properties.

We now turn to the general case. Here, we shall use the following lemma about products in fractional Sobolev spaces. Its proof is presented in Appendix D when $\Omega=\mathbb{R}^{n}$ (see Lemma D.2). The case of a smooth domain $\Omega$ follows by extending the functions to $\mathbb{R}^{n}$. 
Lemma 2. Assume $s \geq 1$ and $1<p<\infty$. Let

$$
f, g \in W^{s, p}(\Omega ; \mathbb{R}) \cap L^{\infty}(\Omega ; \mathbb{R})
$$

where $\Omega$ is a smooth bounded domain in $\mathbb{R}^{n}$. Then

$$
f D g \in W^{s-1, p}(\Omega) .
$$

Proof of Theorem 1 completed. We recall that there is a neighborhood $Q$ of $I \times\{0\}$ in $I^{2}$ and an extension $v$ of $u$ such that

$$
v \in W^{s+(1 / p), p}\left(Q ; S^{1}\right) .
$$

Applying once more the same construction we find some

$$
w \in W^{s+(2 / p), p}\left(U ; S^{1}\right)
$$

where $U$ is a neighborhood of $Q \times\{0\}$ in $Q \times I$. (This construction is possible since $(s+1 / p) p=2$, so that we are again in a limiting case for the Sobolev imbedding and thus $v \in \mathrm{VMO}$. Iterating this construction we find some

$$
\zeta \in W^{s+(k / p), p}\left(G ; S^{1}\right)
$$

where $G$ is a domain in $\mathbb{R}^{k+1}$. Consider the first integer $k \geq 1$ such that

$$
s+(k / p) \geq 1 .
$$

This choice of $k$ implies that

$$
s+\frac{j}{p}<1, \quad j=0,1, \ldots, k-1,
$$

so that, at each step, standard trace theory applies (recall that a function in $W^{s, p}$ has an extension in $W^{s+1 / p, p}$ provided $s$ is not an integer).

¿From the Gagliardo-Nirenberg inequality (see Lemma D.1) we have

$$
\zeta \in W^{1, k+1}\left(G ; S^{1}\right) .
$$

Applying the theorem of Bethuel and Zheng, we may write

$$
\zeta=e^{i \psi}
$$

for some $\psi \in W^{1, k+1}(G ; \mathbb{R})$. Differentiating (2.1) we find

$$
D \psi=-i \bar{\zeta} D \zeta .
$$


By Lemma 2 we have

$$
D \psi \in W^{s+(k / p)-1, p}(G)
$$

and hence

$$
\psi \in W^{s+(k / p), p}(G) .
$$

Taking back traces we obtain

$$
\varphi=\psi_{\left.\right|_{I}} \in W^{s, p}(I)
$$

and

$$
u=e^{i \varphi} .
$$

Remark 3. In one dimension we have established that every $u \in H^{1 / 2}\left(\Omega ; S^{1}\right)$ admits a lifting $\varphi \in H^{1 / 2}\left(\Omega ; S^{1}\right)$. Moreover, this lifting is unique modulo an additive constant (see Appendix B) and the map $u \mapsto \varphi$ is continuous from $H^{1 / 2}$ into $H^{1 / 2}$ (this can be established using the same argument as in Step 7 of the proof of Theorem 4 in Brezis-Nirenberg [1]). Surprisingly there is no bound whatsoever for $\|\varphi\|_{H^{1 / 2}}$ in terms of $\|u\|_{H^{1 / 2}}$. Here is an example of a sequence $\left(\varphi_{n}\right)$ such that $\left\|\varphi_{n}\right\|_{H^{1 / 2}} \rightarrow+\infty$ while $\left\|e^{i \varphi_{n}}\right\|_{H^{1 / 2}} \leq C$. On $\Omega=(-1,+1)$ consider the sequence of functions $\varphi_{n}$ defined by

$$
\varphi_{n}(x)= \begin{cases}0 & \text { for }-1<x<0 \\ 2 \pi n x & \text { for } 0<x<1 / n \\ 2 \pi & \text { for } 1 / n<x<1\end{cases}
$$

Clearly $\left\|\varphi_{n}\right\|_{H^{1 / 2}} \rightarrow+\infty$ (since $\varphi_{n} \rightarrow \varphi=\mathbf{1}_{(0,1)}$ in $L^{2}$ and $\varphi$ does not belong to $H^{1 / 2}$ ). In fact, a more precise computation left to the reader shows that $\left\|\varphi_{n}\right\|_{H^{1 / 2}} \geq c(\log n)^{1 / 2}$ with $c>0$. On the other hand the reader will easily check (for example by scaling) that $\left\|e^{i \varphi_{n}}-1\right\|_{H^{1 / 2}}$ remains bounded. The same conclusion holds when $H^{1 / 2}$ is replaced by $W^{1 / p, p}$ with any $p, 1<p<\infty$.

Remark 4. As we have just pointed out there is no control of $\varphi$ in $H^{1 / 2}$ in terms of $e^{i \varphi}$ in $H^{1 / 2}$. There is, however, (in dimension one), an estimate for $(\varphi-\oint \varphi)$ in the space $H^{1 / 2}+W^{1,1}$, equipped with its usual norm, in terms of $\left\|e^{i \varphi}\right\|_{H^{1 / 2}}$. Here is the argument, working for simplicity with periodic functions. We may also assume (by density) that $\varphi$ is smooth. Observe that the dual of $H^{1 / 2}+W^{1,1}$ is $H^{-1 / 2} \cap W^{-1, \infty}$. Given any $T \in H^{-1 / 2} \cap W^{-1, \infty}$, write $T=\psi^{\prime}+c$ for some $\psi \in H^{1 / 2} \cap L^{\infty}$ and some constant $c$. Then

$$
\langle T, \varphi-\oint \varphi\rangle=\left\langle\psi^{\prime}, \varphi-\oint \varphi\right\rangle=-\left\langle\psi, \varphi^{\prime}\right\rangle .
$$

But if we set $u=e^{i \varphi}$, then $\varphi^{\prime}=-i \bar{u} u^{\prime}$ and thus

$$
|\langle T, \varphi-\oint \varphi\rangle|=\left|\left\langle\psi, i \bar{u} u^{\prime}\right\rangle\right|=\left|\left\langle u^{\prime}, i \psi \bar{u}\right\rangle\right| \leq\|u\|_{H^{1 / 2}}\|\psi u\|_{H^{1 / 2}} .
$$


Recall that $H^{1 / 2} \cap L^{\infty}$ is an algebra (see e.g. Appendix D) and that

$$
\begin{aligned}
\|\psi u\|_{H^{1 / 2}} & \leq C\left(\|\psi\|_{H^{1 / 2}}+\|\psi\|_{L^{\infty}}\right)\left(\|u\|_{H^{1 / 2}}+\|u\|_{L^{\infty}}\right) \\
& \leq C\|T\|_{H^{-1 / 2} \cap W^{-1, \infty}}\left(\|u\|_{H^{1 / 2}}+1\right) .
\end{aligned}
$$

We conclude that

$$
\|\varphi-\oint \varphi\|_{H^{1 / 2}+W^{1,1}} \leq C\|u\|_{H^{1 / 2}}\left(\|u\|_{H^{1 / 2}}+1\right) .
$$

The same estimate holds in higher dimensions if $u$ belongs to the closure of $C^{\infty}\left(\bar{\Omega} ; S^{1}\right)$ in $H^{1 / 2}\left(\Omega ; S^{1}\right)$; however, the argument is much more delicate and will be presented in our forthcoming paper, Bourgain, Brezis and Mironescu [1].

\section{Proof of Theorem 3 when $s p \geq 2$.}

The case $s=1$ in Theorem 3 coincides with the theorem of Bethuel and Zheng. For the sake of completeness we present a proof which is simpler than the original one (see also Carbou [1] for a similar idea).

Proof of the Bethuel-Zheng theorem. The idea is to assume that $\varphi$ is known and to derive some consequences. Writing $u=u_{1}+i u_{2} \quad$ with $u_{1}=\cos \varphi$ and $u_{2}=\sin \varphi$ we have

$$
D u_{1}=-(\sin \varphi) D \varphi=-u_{2} D \varphi
$$

and

$$
D u_{2}=(\cos \varphi) D \varphi=u_{1} D \varphi
$$

Hence

$$
D \varphi=u_{1} D u_{2}-u_{2} D u_{1}
$$

The strategy is now to find $\varphi$ by solving (3.1) with the help of a generalized form of Poincaré's lemma,

Lemma 3. Let $1 \leq p<\infty$ and let $f \in L^{p}\left(\Omega ; \mathbb{R}^{n}\right)$. The following properties are equivalent:

a) there is some $\varphi \in W^{1, p}(\Omega ; \mathbb{R})$ such that

$$
f=D \varphi
$$

b) one has

$$
\frac{\partial f_{i}}{\partial x_{j}}=\frac{\partial f_{j}}{\partial x_{i}} \quad \forall i, j, \quad 1 \leq i, j \leq n
$$


in the sense of distributions, i.e.,

$$
\int f_{i} \frac{\partial \psi}{\partial x_{j}}=\int f_{j} \frac{\partial \psi}{\partial x_{i}} \quad \forall \psi \in C_{0}^{\infty}(\Omega) .
$$

We emphasize that the assumption that $\Omega$ is simply connected is needed in this lemma.

Proof of Lemma 3. The implication $a) \Rightarrow b$ ) is obvious. To prove the converse, let $\bar{f}$ be the extension of $f$ by 0 outside $\Omega$ and let $\bar{f}_{\varepsilon}=\rho_{\varepsilon} \star \bar{f}$ where $\left(\rho_{\varepsilon}\right)$ is a sequence of mollifiers. The $\bar{f}_{\varepsilon}$ 's satisfy (3.2) on every compact subset of $\Omega$ (for $\varepsilon$ sufficiently small). In particular, on every smooth simply connected domain $\omega \subset \Omega$ with compact closure in $\Omega$, there is a function $\psi_{\varepsilon}$ such that

$$
D \psi_{\varepsilon}=\bar{f}_{\varepsilon} \quad \text { in } \omega .
$$

(Here we have used the standard Poincaré lemma). Passing to the limit we obtain some $\psi \in W^{1, p}(\omega)$ such that $D \psi=f$ in $\omega$. Finally, we write $\Omega$ as an increasing union of $\omega_{n}$ as above and obtain a corresponding sequence $\psi_{n}$. In the limit we find some $\varphi \in L_{\text {loc }}^{1}(\Omega)$ with $D \varphi=f$ in $\Omega$. Using the regularity of $\Omega$ and a standard property of Sobolev spaces (see e.g. Maz'ja [1], Corollary in Section 1.1.11) we conclude that $\varphi \in W^{1, p}(\Omega)$.

Proof of the Bethuel-Zheng theorem completed. We will first verify condition $b$ ) of the lemma for

$$
f=u_{1} D u_{2}-u_{2} D u_{1}
$$

i.e.,

$$
f_{i}=u_{1} \frac{\partial u_{2}}{\partial x_{i}}-u_{2} \frac{\partial u_{1}}{\partial x_{i}}
$$

Formally, property (3.2) is clear. Indeed, if $u_{1}$ and $u_{2}$ are smooth, then

$$
\frac{\partial f_{i}}{\partial x_{j}}-\frac{\partial f_{j}}{\partial x_{i}}=2\left(\frac{\partial u_{1}}{\partial x_{j}} \frac{\partial u_{2}}{\partial x_{i}}-\frac{\partial u_{1}}{\partial x_{i}} \frac{\partial u_{2}}{\partial x_{j}}\right)
$$

On the other hand, if we differentiate the relation

$$
|u|^{2}=u_{1}^{2}+u_{2}^{2}=1
$$

we find

$$
u_{1} \frac{\partial u_{1}}{\partial x_{i}}+u_{2} \frac{\partial u_{2}}{\partial x_{i}}=0 \quad \forall i=1,2, \ldots, n
$$

Thus, in $\mathbb{R}^{2}$, the vector $\left(\frac{\partial u_{1}}{\partial x_{i}}, \frac{\partial u_{2}}{\partial x_{i}}\right)$ is orthogonal to $\left(u_{1}, u_{2}\right)$. It follows that the vectors $\left(\frac{\partial u_{1}}{\partial x_{i}}, \frac{\partial u_{2}}{\partial x_{i}}\right)$ and $\left(\frac{\partial u_{1}}{\partial x_{j}}, \frac{\partial u_{2}}{\partial x_{j}}\right)$ are colinear and therefore

$$
\operatorname{det}\left(\begin{array}{ll}
\frac{\partial u_{1}}{\partial x_{i}} & \frac{\partial u_{2}}{\partial x_{i}} \\
\frac{\partial u_{1}}{\partial x_{j}} & \frac{\partial u_{2}}{\partial x_{j}}
\end{array}\right)=\frac{\partial u_{1}}{\partial x_{i}} \frac{\partial u_{2}}{\partial x_{j}}-\frac{\partial u_{1}}{\partial x_{j}} \frac{\partial u_{2}}{\partial x_{i}}=0 .
$$


Hence (3.2) holds. To make this argument rigorous we rely on the density of smooth functions in the Sobolev space $W^{1, p}(\Omega ; \mathbb{R})$ (see e.g. Adams [1], Chap. III or Brezis [1], Chap. IX): there are sequences $\left(u_{1 n}\right)$ and $\left(u_{2 n}\right)$ in $C^{\infty}(\bar{\Omega} ; \mathbb{R})$ such that $u_{1 n} \rightarrow u_{1}$ and $u_{2 n} \rightarrow u_{2}$ in $W^{1, p}(\Omega ; \mathbb{R})$ and $\left\|u_{1 n}\right\|_{L^{\infty}} \leq 1,\left\|u_{2 n}\right\|_{L^{\infty}} \leq 1$.

[Warning: We do not claim that $u_{n}=\left(u_{1 n}, u_{2 n}\right)$ takes its values in $S^{1}$. The density of $C^{\infty}(\bar{\Omega} ; N)$ in $W^{1, p}(\Omega ; N)$, where $N$ is a compact manifold without boundary, e.g. $N=S^{1}$, is a delicate matter which has been extensively studied by Bethuel [1]. As a matter of fact, the Bethuel-Zheng theorem can be used to prove the density of $C^{\infty}\left(\bar{\Omega} ; S^{1}\right)$ in $W^{1, p}\left(\Omega ; S^{1}\right)$ for $p \geq 2$.]

Set

$$
f_{n}=u_{1 n} D u_{2 n}-u_{2 n} D u_{1 n}
$$

so that

$$
f_{n} \rightarrow f \quad \text { in } L^{p}
$$

and

$$
\frac{\partial f_{i n}}{\partial x_{j}}-\frac{\partial f_{j n}}{\partial x_{i}}=2\left(\frac{\partial u_{1 n}}{\partial x_{j}} \frac{\partial u_{2 n}}{\partial x_{i}}-\frac{\partial u_{1 n}}{\partial x_{i}} \frac{\partial u_{2 n}}{\partial x_{j}}\right)
$$

converges in $L^{p / 2}$ to $2\left(\frac{\partial u_{1}}{\partial x_{j}} \frac{\partial u_{2}}{\partial x_{i}}-\frac{\partial u_{1}}{\partial x_{i}} \frac{\partial u_{2}}{\partial x_{j}}\right)$. Multiplying (3.6) by $\psi \in C_{0}^{\infty}(\Omega)$, integrating by parts and passing to the limit (using the fact that $p \geq 2$ ) we obtain

$$
-\int_{\Omega}\left(f_{i} \frac{\partial \psi}{\partial x_{j}}-f_{j} \frac{\partial \psi}{\partial x_{i}}\right)=2 \int_{\Omega}\left(\frac{\partial u_{1}}{\partial x_{j}} \frac{\partial u_{2}}{\partial x_{i}}-\frac{\partial u_{1}}{\partial x_{i}} \frac{\partial u_{2}}{\partial x_{j}}\right) \psi .
$$

On the other hand (3.4) and (3.5) hold a.e. (even for any $u \in W^{1, p}\left(\Omega ; S^{1}\right), 1 \leq p<\infty$ ) It follows that $f$ satisfies $b$ ) of Lemma 3 , and therefore there is some $\varphi \in W^{1, p}(\Omega ; \mathbb{R})$ such that

$$
f=D \varphi .
$$

We will now prove that this $\varphi$ is essentially the one in the conclusion of the Bethuel-Zheng theorem.

Recall that if $g, h \in W^{1, p}(\Omega) \cap L^{\infty}(\Omega)$ with $1 \leq p<\infty$, then $g h \in W^{1, p}$ and

$$
\frac{\partial}{\partial x_{i}}(g h)=g \frac{\partial h}{\partial x_{i}}+h \frac{\partial g}{\partial x_{i}} .
$$

Set

$$
v=u e^{-i \varphi},
$$

so that $v \in W^{1, p}$ and

$$
\begin{aligned}
D v & =e^{-i \varphi}(D u-i D \varphi)=u e^{-i \varphi}(\bar{u} D u-i D \varphi) \\
& =u e^{-i \varphi}(\bar{u} D u-i f)=u e^{-i \varphi}\left(u_{1} D u_{1}+u_{2} D u_{2}\right)=0 \quad \text { by (3.4). }
\end{aligned}
$$


We deduce that $v$ is a constant and since $|v|=1$ we may write $v=e^{i C}$ for some constant $C \in \mathbb{R}$. Hence $u=e^{i(\varphi+C)}$ and the function $\varphi+C$ has the desired properties.

We now turn to the proof of Theorem 3 when $s p \geq 2$. In fact, we have a more precise statement:

Lemma 4. Assume $n \geq 1, s \geq 1,1<p<\infty$ and $s p \geq 2$. Then any $u \in W^{s, p}\left(\Omega ; S^{1}\right)$ may be lifted as $u=e^{i \varphi}$ with $\varphi \in W^{s, p}(\Omega ; \mathbb{R}) \cap W^{1, s p}(\Omega ; \mathbb{R})$.

Proof. Observe that

$$
W^{s, p} \cap L^{\infty} \subset W^{1, s p}
$$

by the Gagliardo-Nirenberg inequality (see Lemma D.1). Since $s p \geq 2$ we may apply the Bethuel-Zheng theorem and write $u=e^{i \varphi}$ for some $\varphi \in W^{1, s p}(\Omega ; \mathbb{R})$. Using Lemma 2 we find that

$$
D \varphi=-i \bar{u} D u \in W^{s-1, p}
$$

so that $\varphi \in W^{s, p}$.

\section{Examples of obstruction in Theorems 2 and 3.}

We start with an example of obstruction in Theorem 2, i.e., when $0<s<1$ and $1 \leq s p<n$.

Lemma 5. Assume $n \geq 2$. Given any $s$ and any $p$ with $0<s<1,1<p<\infty$, and $1 \leq s p<n$, there is some $u \in W^{s, p}\left(\Omega ; S^{1}\right)$ which cannot be lifted, i.e., for this $u$ no $\varphi \in W^{s, p}(\Omega ; \mathbb{R})$ exists such that $u=e^{i \varphi}$.

Proof. Without loss of generality we may assume that $\Omega$ is the unit ball. Let

$$
\psi(x)=\frac{1}{|x|^{\alpha}} \quad \text { with } \frac{n-s p}{p} \leq \alpha<\frac{n-s p}{s p}
$$

and let

$$
u=e^{i \psi}
$$

We claim that

$$
u \in W^{s, p}\left(\Omega ; S^{1}\right) .
$$

Indeed it is clear that

$$
\psi \in W^{1, q} \quad \forall q \text { with } 1<q<\frac{n}{\alpha+1}
$$

and thus

$$
\psi \in W^{\sigma, q} \quad \forall \sigma \text { with } 0<\sigma<1, \quad \forall q \text { with } 1<q<\frac{n}{\alpha+1} \text {. }
$$


Since $u \in L^{\infty}$, we also know, by the Gagliardo-Nirenberg inequality (see Lemma D.1 in Appendix D), that

$$
u \in W^{t, r} \forall t \in(0,1) \forall r \in(1, \infty) \quad \text { with } t r<\frac{n}{\alpha+1}
$$

In particular, we may choose $t=s$ and $r=p$ since $s p<n /(\alpha+1)$, i.e., (4.1) holds.

Next we claim that there is no $\varphi \in W^{s, p}(\Omega ; \mathbb{R})$ such that $u=e^{i \varphi}$. Assume, by contradiction, that such $\varphi$ exists. Set

$$
\eta=\frac{1}{2 \pi}(\varphi-\psi)
$$

so that $\eta$ takes its values in $\mathbb{Z}$ and

$$
\eta \in W_{\text {loc }}^{s, p}(\Omega \backslash\{0\} ; \mathbb{Z})
$$

(because $\psi$ is smooth on $\Omega \backslash\{0\}$ ). Since $s p \geq 1$ and $\Omega \backslash\{0\}$ is connected we conclude, using Lemma B.1 in Appendix B, that $\eta$ is a constant. Thus $\psi \in W^{s, p}(\Omega ; \mathbb{R})$. Note that, by scaling,

$$
A(r)=\int_{B_{r}} \int_{B_{r}} \frac{|\psi(x)-\psi(y)|^{p}}{|x-y|^{n+s p}} d x d y
$$

satisfies $A(1)=r^{\beta} A(r)$ with $\beta=(\alpha+s) p-n \geq 0$ (by assumption on $\alpha$ ). If $A(1)<\infty$, then $A(1)=0$ (by letting $r \rightarrow 0$ ). But this is impossible. Thus $A(1)=\infty$, i.e., $\psi \notin W^{s, p}$. A contradiction.

A topological obstruction. There is an alternative example of obstruction to lifting, which is of topological nature.

Consider first the case $n=2$. Set

$$
u(x)=\frac{x}{|x|} \quad \text { on the unit ball } \Omega \text { of } \mathbb{R}^{2} .
$$

Since

$$
|D u(x)| \leq C /|x|
$$

we see that $u \in W^{1, q}\left(\Omega ; S^{1}\right)$ for every $q<2$ and therefore $u \in W^{s, p}\left(\Omega ; S^{1}\right)$ for every $s \in(0,1)$ and every $p \in(1, \infty)$ with $s p<2$ (by the Gagliardo-Nirenberg inequality; see Lemma D.1), If, in addition, we assume $s p \geq 1$ then there is no $\varphi \in W^{s, p}(\Omega ; \mathbb{R})$ such that $u=e^{i \varphi}$. Indeed set

$$
\Omega^{\prime}=\Omega \backslash([0,1] \times\{0\})
$$

and

$$
\theta \in(0,2 \pi) \quad \text { with } e^{i \theta}=u .
$$

Clearly $\theta \in C^{\infty}\left(\Omega^{\prime}\right)$ and $\theta$ has a jump of $2 \pi$ along the segment $[0,1] \times\{0\}$. Assume, by contradiction, that $u$ has a lifting $\varphi \in W^{s, p}(\Omega ; \mathbb{R})$. Arguing as above we would conclude 
that $\theta \in W^{s, p}(\Omega ; \mathbb{R})$ but this is impossible since $\theta$ has a jump of $2 \pi$ along the segment $(0,1) \times\{0\}$ and such a function cannot belong to $W^{s, p}$ with $s p \geq 1$.

When $n \geq 3$, the same construction as above with

$$
u(x)=\frac{\left(x_{1}, x_{2}\right)}{\left(x_{1}^{2}+x_{2}^{2}\right)^{1 / 2}} \quad x=\left(x_{1}, x_{2}, \ldots, x_{n}\right)
$$

provides an example of a function $u \in W^{s, p}\left(\Omega ; S^{1}\right)$ for every $s \in(0,1)$ and every $p \in(1, \infty)$ with $s p<2$ and which has no lifting in $W^{s, p}$ when $s p \geq 1$. However, this example does not reach the optimal condition $s p<n$ when $n \geq 3$.

Remark 5. The topological obstruction provides an example of loss of regularity in lifting. To explain the phenomenon consider the simple case where $p=2$. Recall (see Corollary 1) that if $u \in H^{s}\left(\Omega ; S^{1}\right)$ with $1 / 2<s<1$, then, in general, $u$ has no lifting in $H^{s}$. From the positive part in Corollary 1 one knows that $u$ has a lifting in $H^{(1 / 2-\varepsilon)}$. Roughly speaking, we lose $(s-1 / 2)$ derivative in the lifting.

Open Problem: When $n \geq 3$ the precise loss of regularity in lifting is not fully understood. For simplicity consider the case $n=3$ and $p=4$. First a summary of the known results:

a) If $s<1 / 4$, any $u \in W^{s, 4}$ has a lifting in $W^{s, 4}$.

b) If $s \geq 3 / 4$, any $u \in W^{s, 4}$ has a lifting in $W^{s, 4}$.

c) If $1 / 4 \leq s<3 / 4$ some $u$ 's in $W^{s, 4}$ have no lifting in $W^{s, 4}$.

d) The topological example provides an example of a function $u \in W^{s, 4} \forall s<1 / 2$, and this $u$ has no lifting even in $W^{1 / 4,4}$.

It would be interesting to find an example of a function $u \in W^{s, 4} \forall s<3 / 4$ which has no lifting even in $W^{1 / 4,4}$.

Finally, case $b$ ) in Theorem 3 relies on

Lemma 6. Assume $n \geq 2$. Given any $s$ and any $p$ with $s \geq 1$ and $1<p<\infty$ with $s p<2$, there is some $u \in W^{s, p}\left(\Omega ; S^{1}\right)$ which cannot be lifted by a function $\varphi \in W^{s, p}(\Omega ; \mathbb{R})$.

Proof. Use the topological example $u$ above. It is easy to see that $u \in W^{s, p} \forall s \in(0, \infty)$, $\forall p \in(1, \infty)$ with $s p<2$. This $u$ has no lifting even in $W^{1 / p, p}$.

\section{Control of lifting in the $H^{s}$-norm for $s \overrightarrow{<} \frac{1}{2}$ and application to Ginzburg- Landau.}

We return to the particular issue of lifting a function $u \in H^{s}\left(\Omega ; S^{1}\right)$ when $s<1 / 2$ and $s \rightarrow 1 / 2$. Recall (see Corollary 1 ) that, for every $s<1 / 2, u$ admits a lifting $\varphi \in H^{s}(\Omega ; \mathbb{R}$ ), i.e.,

$$
u=e^{i \varphi}
$$


We also know (see (1.7)) that we may find a $\varphi \in H^{s}$ such that

$$
\|\varphi\|_{H^{s}} \leq C_{s}\|u\|_{H^{s}}
$$

Our aim is to find an optimal control for the constant $C_{s}$ as $s \rightarrow 1 / 2$. Such a control will then be used in the study of the Ginzburg-Landau energy $E_{\varepsilon}$ as $\varepsilon \rightarrow 0$.

If we follow the proof in Section 1 we obtain a $\varphi$ as a limit of sequence $\varphi_{j}$ such that

$$
\sum_{j \geq 1} 4^{s j}\left\|\varphi_{j}-\varphi_{j-1}\right\|_{L^{2}}^{2} \leq C \sum_{j \geq 0} 4^{s j}\left\|u-E_{j}(u)\right\|_{L^{2}}^{2}
$$

where here, and in what follows, $C$ without a subscript $s$ denotes a constant which remains bounded as $s \rightarrow 1 / 2$. Following the proof of Corollary 1 we obtain

$$
\sum_{j \geq 1} 4^{s j}\left\|E_{j}(\varphi)-E_{j-1}(\varphi)\right\|_{L^{2}}^{2} \leq C \sum_{j \geq 1} 4^{s j}\left\|\varphi_{j}-\varphi_{j-1}\right\|_{L^{2}}^{2}
$$

We also recall (see Step 3 in Appendix A) that

$$
\sum_{j \geq 0} 4^{s j}\left\|u-E_{j}(u)\right\|_{L^{2}}^{2} \leq C\|u\|_{H^{s}}^{2} .
$$

Combining (5.2), (5.3) and (5.4) yields

$$
\sum_{j \geq 1} 4^{s j}\left\|E_{j}(\varphi)-E_{j-1}(\varphi)\right\|_{L^{2}}^{2} \leq C\|u\|_{H^{s}}^{2}
$$

Finally we know (see Corollary A.2 in Appendix A) that

$$
\|\varphi\|_{H^{s}} \leq C_{s}\left(\sum_{j \geq 1} 4^{s j}\left\|E_{j}(\varphi)-E_{j-1}(\varphi)\right\|_{L^{2}}^{2}\right)^{1 / 2}
$$

and the optimal constant $C_{s}$ for the inequality (5.6) is of the order of $(1-2 s)^{-1}$. Hence we deduce that the $\varphi$ constructed by this technique satisfies

$$
\|\varphi\|_{H^{s}} \leq C(1-2 s)^{-1}\|u\|_{H^{s}} .
$$

In fact, there is a more refined construction of lifting which yields a better estimate. For simplicity we work in a cube $Q$ of $\mathbb{R}^{d}, d \geq 1$; for more general domains see Remark E.2 in Appendix E. 
Theorem 4. For every $u \in H^{s}\left(Q ; S^{1}\right)$ with $0<s<1 / 2$ one may construct a $\varphi \in$ $H^{s}(Q ; \mathbb{R})$ satisfying (5.1) and the (optimal) estimate

$$
\|\varphi\|_{H^{s}} \leq C(1-2 s)^{-1 / 2}\|u\|_{H^{s}}
$$

where $C$ is independent of $u$ and independent of $s$ as $s \rightarrow 1 / 2$.

The reason why the previous construction does not yield the correct asymptotic as $s \rightarrow 1 / 2$ is due to "edge-singularities" at the nodes of our dyadic partitions $P_{j}$. To overcome this, we rely on an argument of translations which is explained in Appendix E where we present the proof of Theorem 4. That type of argument has been exploited earlier in slightly different contexts (for instance in comparing the usual and dyadic BMO-norms, see Garnett and Jones [1]).

The next result is an application to the Ginzburg-Landau functional. Let $Q$ be a cube of $\mathbb{R}^{d}, d \geq 1$, and let $\Omega=Q \times(0,1)$. For any function $g \in H^{1 / 2}(Q ; \mathbb{C})$ set

$$
\begin{aligned}
H_{g}^{1}(\Omega) & =\left\{u(x, t): \Omega \rightarrow \mathbb{C} ; \int_{\Omega}|\nabla u|^{2} d x d t<\infty \text { and } u(x, 0)=g(x) \text { on } Q\right\}, \\
E_{\varepsilon}(u) & =\frac{1}{2} \int_{\Omega}|\nabla u|^{2}+\frac{1}{4 \varepsilon^{2}} \int_{\Omega}\left(|u|^{2}-1\right)^{2},
\end{aligned}
$$

where $\nabla$ denotes the full gradient (in $(x, t))$.

Theorem 5. For every $g \in H^{1 / 2}\left(Q ; S^{1}\right)$ we have, for $\varepsilon>0$,

$$
E_{\varepsilon}=\operatorname{Min}_{u \in H_{g}^{1}(\Omega)} E_{\varepsilon}(u) \leq C \log (1 / \varepsilon)\|g\|_{H^{1 / 2}}^{2}
$$

where $C$ is independent of $\varepsilon$ and of $g$.

Proof. Let $s=s(\varepsilon)<1 / 2$ to be specified. It follows from Theorem 4 (applied to $g$ ) that $g=e^{i \varphi}$ for some $\varphi \in H^{s}(Q ; \mathbb{R})$ satisfying

$$
\|\varphi\|_{H^{s}} \leq C(1-2 s)^{-1 / 2}\|g\|_{H^{1 / 2}} .
$$

Denote $\varphi_{\delta}$ a $\delta$-smoothing of $\varphi$ (with $\delta$ to be chosen later). Thus, we have

$$
\left\|\varphi-\varphi_{\delta}\right\|_{L^{2}(Q)} \leq C \delta^{s}\|\varphi\|_{H^{s}(Q)} \leq C \delta^{s}(1-2 s)^{-1 / 2}\|g\|_{H^{1 / 2}(Q)}
$$

also, by (5.10),

$$
\left\|\varphi_{\delta}\right\|_{H^{1 / 2}(Q)} \leq C \delta^{s-1 / 2}\|\varphi\|_{H^{s}(Q)} \leq C(1-2 s)^{-1 / 2} \delta^{s-1 / 2}\|g\|_{H^{1 / 2}(Q)} .
$$

Taking

$$
1-2 s \sim(\log 1 / \delta)^{-1}
$$


we conclude that

$$
\left\|\varphi_{\delta}\right\|_{H^{1 / 2}(Q)} \leq C(\log 1 / \delta)^{1 / 2}\|g\|_{H^{1 / 2}(Q)}
$$

Let $\tilde{\varphi}_{\delta}$ denote some harmonic extension of $\varphi_{\delta}$ to $\Omega$ with

$$
\left\|\tilde{\varphi}_{\delta}\right\|_{H^{1}(\Omega)} \leq C(\log 1 / \delta)^{1 / 2}\|g\|_{H^{1 / 2}(Q)}
$$

and set

$$
G_{\delta}=e^{i \tilde{\varphi} \delta}
$$

so that

$$
\left\|G_{\delta}\right\|_{H^{1}(\Omega)} \leq C(\log 1 / \delta)^{1 / 2}\|g\|_{H^{1 / 2}(Q)} .
$$

Let $P$ denote some harmonic extension of $\left(g-e^{i \varphi_{\delta}}\right)$ to $\Omega$ satisfying the following three estimates

$$
\begin{aligned}
&\|P\|_{H^{1}(\Omega)} \leq C\left\|g-e^{i \varphi_{\delta}}\right\|_{H^{1 / 2}(Q)} \\
& \leq C\left(\|g\|_{H^{1 / 2}(Q)}+\left\|\varphi_{\delta}\right\|_{H^{1 / 2}(Q)}\right) \\
& \leq C(\log 1 / \delta)^{1 / 2}\|g\|_{H^{1 / 2}(Q)} \text { by }(5.14) \\
&\|P\|_{L^{\infty}(\Omega)} \leq C\left\|g-e^{i \varphi_{\delta}}\right\|_{L^{\infty}(Q)} \leq C
\end{aligned}
$$

and

$$
\begin{aligned}
\|P\|_{L^{2}(\Omega)} & \leq C\left\|g-e^{i \varphi_{\delta}}\right\|_{L^{2}(Q)} \\
& \leq C\left\|\varphi-\varphi_{\delta}\right\|_{L^{2}(Q)} \leq C \delta^{1 / 2}(\log 1 / \delta)^{1 / 2}\|g\|_{H^{1 / 2}(Q)} \text { by }(5.11) .
\end{aligned}
$$

Define

$$
u=G_{\delta}+P
$$

so that by construction $u_{\mid t=0}=g$ on $Q$.

¿From (5.17) and (5.18) we have

$$
\|u\|_{H^{1}(\Omega)}^{2} \leq C \log (1 / \delta)\|g\|_{H^{1 / 2}(Q)}^{2} .
$$


On the other hand, using (5.19) we find

$$
\left.|| u\right|^{2}-1|\leq C||u|-1|||u|+1|\leq C||u|-1 \mid
$$

and since

$$
|| u|-1|=|| u|-| G_{\delta}|| \leq\left|u-G_{\delta}\right|=|P|
$$

we are led to

$$
\int_{\Omega}\left(|u|^{2}-1\right)^{2} \leq C \int_{\Omega}|P|^{2} \leq C \delta(\log 1 / \delta)\|g\|_{H^{1 / 2}(Q)} \text { by }(5.20) .
$$

Combining (5.22) and (5.23) we obtain

$$
E_{\varepsilon}(u) \leq C\left(1+\delta / \varepsilon^{2}\right) \log (1 / \delta)\|g\|_{H^{1 / 2}(Q)}^{2} .
$$

Choosing $\delta=\varepsilon^{2}$ yields the desired estimate (5.9).

Remark 6. In dimension $d=1, E_{\varepsilon}$ remains bounded as $\varepsilon \rightarrow 0$ since we may write $g=e^{i \varphi}$ with some $\varphi \in H^{1 / 2}$ and then take $u=e^{i \tilde{\varphi}}$ where $\tilde{\varphi}$ is some harmonic extension of $\varphi$. However, the bound for $E_{\varepsilon}$ depends on $g$, not just on $\|g\|_{H^{1 / 2}}$ (see also Remark 3).

Remark 7. In dimension $d \geq 2$, estimate (5.9) is optimal. This may be seen, for example in dimension $d=2$, by choosing for $g$ the topological example described in Section 4 ,

$$
g(x)=\frac{x}{|x|} \text { on } Q
$$

We claim that $E_{\varepsilon} \geq \alpha \log (1 / \varepsilon)$ for some constant $\alpha>0$. Indeed we may write for any $u \in H_{g}^{1}(\Omega)$,

$$
E_{\varepsilon}(u) \geq \alpha \int_{1 / 2}^{1} d r \int_{\sum_{r}}\left(\frac{1}{2}\left|\nabla_{\sigma} u\right|^{2}+\frac{1}{4 \varepsilon}\left(|u|^{2}-1\right)^{2}\right) d \sigma
$$

where $\Sigma_{r}=\left\{(x, t) \in \Omega ;|x|^{2}+t^{2}=r^{2}\right\}$ and $\nabla_{\sigma}$ denote the tangential gradient on $\Sigma_{r}$. We then invoke the lower bound

$$
\frac{1}{2} \int_{\sum_{r}}\left|\nabla_{\sigma} u\right|^{2}+\frac{1}{4 \varepsilon^{2}} \int_{\sum_{r}}\left(|u|^{2}-1\right)^{2} \geq c(\log 1 / \varepsilon)
$$

which is known for a 2-dimensional flat disk (see Bethuel, Brezis and Hélein [1], Theorem V.3) and can be transported to $\Sigma_{r}$ by a smooth diffeomorphism.

The fact that (5.9) is optimal when $d \geq 2$ shows in turn that (5.8) is also optimal for $d \geq 2$. Indeed an estimate of the form $\|\varphi\|_{H^{s}} \leq o\left((1-2 s)^{-1 / 2}\right)$ in place of (5.8), would yield $E_{\varepsilon} \leq o(\log 1 / \varepsilon)$, which is impossible. When $d=1$, estimate (5.8) is still optimal, but this requires a separate argument (see Remark E.1 in Appendix E). 
Remark 8. Theorem 4 is still valid for a general smooth domain $Q$ in $\mathbb{R}^{d}$ (without any topological assumption); see Remark E.2 in Appendix E. As a result, Theorem 5 is also true in that situation. In Theorem 5 we may also take for $\Omega$ any smooth bounded domain in $\mathbb{R}^{d+1}, d \geq 1$ and $Q=\partial \Omega$; this is a consequence of the fact that Theorem 4 is still valid when $Q$ is a smooth $d$-dimensional manifold (see Remark E.2 in Appendix E). In that case a more elementary (and simple) proof of (5.9) was obtained recently by $\mathrm{T}$. Rivière [3]. Estimate (5.9) plays a fundamental role in the asymptotic analysis (as $\varepsilon \rightarrow 0$ ) of Ginzburg-Landau minimizers (see Rivière [1], [2], Lin and Rivière [1], Sandier [1] and also the forthcoming paper Bourgain, Brezis and Mironescu [1]).

\section{APPENDIX A}

\section{A characterization of $\mathbf{W}^{\mathbf{s}, p}(\Omega ; \mathbb{R})$ when $\mathbf{s p}<\mathbf{1}$}

Let $\Omega=(0,1)^{n}$. For $j=0,1, \ldots$ we denote by $\mathcal{P}_{j}$ the dyadic partition of $\Omega$ into $2^{j n}$ cubes of side $2^{-j}$ and by $\mathcal{E}_{j}$ the space of functions from $\Omega$ into $\mathbb{R}$ (or $\mathbb{C}$ ) which are constant on each cube of $\mathcal{P}_{j}$. Given a function $f \in L^{p}(\Omega)$ we consider the function $f_{j}=E_{j}(f) \in \mathcal{E}_{j}$ defined as follows: every $x \in \Omega$ belongs to one of the cubes, say $Q_{j}(x)$, of the partition $\mathcal{P}_{j}$ and we set

$$
f_{j}(x)=E_{j}(f)(x)=\oint_{Q_{j}(x)} f .
$$

Clearly we have

$$
\begin{gathered}
\left\|E_{j}(f)\right\|_{L^{p}} \leq\|f\|_{L^{p}} \quad \forall j, \\
E_{j}(f) \rightarrow f \quad \text { in } L^{p} \text { and a.e. as } j \rightarrow \infty .
\end{gathered}
$$

Theorem A.1. Assume $s p<1$. Then

$$
\begin{aligned}
\|f\|_{W^{s, p}}^{p} & \sim \sum_{j \geq 1} 2^{s p j}\left\|E_{j}(f)-E_{j-1}(f)\right\|_{L^{p}}^{p} \\
& \sim \sum_{j \geq 0} 2^{s p j}\left\|f-E_{j}(f)\right\|_{L^{p}}^{p} .
\end{aligned}
$$

Remark A.1. Theorem A.1 is due to G. Bourdaud [1] (see his Théorème 5 with $m=0$ and also the earlier paper of R. Devore and V. A. Popov [1]). It gives a positive answer to a conjecture of $\mathrm{H}$. Triebel [1] (Conjecture 1 ) for the Haar system $\left\{h_{j}^{(-1,0)}\right\}$ in the spaces $B_{p, p}^{s}=W^{s, p}$. The parameter $\ell=-1+1-0=0$ and (for $s>0$ ), the condition $s<\ell+(1 / p)$ is indeed $s p<1$. For the convenience of the reader, and also because we are interested in the behaviour of the sharp constants in the norm equivalence as $s p \rightarrow 1$, we present below a proof of Theorem A.1.

We have also made use of the 
Corollary A.1. Assume $s p<1$ and let $\left(\varphi_{j}\right)_{j=0,1, \ldots}$ be a sequence of functions on $\Omega$ such that

$$
\varphi_{j} \in \mathcal{E}_{j} \quad \forall j=0,1 \ldots
$$

and

$$
\sum_{j \geq 1} 2^{s p j}\left\|\varphi_{j}-\varphi_{j-1}\right\|_{L^{p}}^{p}<\infty
$$

Then $\varphi_{j} \rightarrow \varphi$ in $L^{p}$ and $\varphi \in W^{s, p}$ with

$$
\|\varphi\|_{W^{s, p}}^{p} \leq C \sum_{j \geq 1} 2^{s p j}\left\|\varphi_{j}-\varphi_{j-1}\right\|_{L^{p}}^{p} .
$$

Remark A.2. Here $\|f\|_{W^{s, p}}$ denotes the standard semi-norm,

$$
\|f\|_{W^{s, p}}^{p}=\int_{\Omega} \int_{\Omega} \frac{|f(x)-f(y)|^{p}}{|x-y|^{n+s p}} d x d y .
$$

To work with a norm it suffices to add $\left|\int f\right|$.

Proof of Corollary A.1. From (A.4) we see that $\varphi_{j}$ is a Cauchy sequence in $L^{p}$ and thus $\varphi_{j} \rightarrow \varphi$ in $L^{p}$. In order to prove that $\varphi \in W^{s, p}$ it suffices, in view of Theorem A.1, to check that

$$
\sum_{j \geq 1} 2^{s p j}\left\|E_{j}(\varphi)-E_{j-1}(\varphi)\right\|_{L^{p}}^{p}<\infty
$$

Note that

$$
E_{j}(\varphi)-E_{j-1}(\varphi)=E_{j}\left(\varphi-\varphi_{j}\right)-E_{j-1}\left(\varphi-\varphi_{j-1}\right)+\varphi_{j}-\varphi_{j-1}
$$

and thus

$$
\left\|E_{j}(\varphi)-E_{j-1}(\varphi)\right\|_{L^{p}} \leq\left\|\varphi-\varphi_{j}\right\|_{L^{p}}+\left\|\varphi-\varphi_{j-1}\right\|_{L^{p}}+\left\|\varphi_{j}-\varphi_{j-1}\right\|_{L^{p}}
$$

On the other hand, if we write

$$
\varphi_{j}-\varphi=\left(\varphi_{j}-\varphi_{j+1}\right)+\left(\varphi_{j+1}-\varphi_{j+2}\right)+\cdots,
$$

we see that

$$
\left\|\varphi_{j}-\varphi\right\|_{L^{p}} \leq \sum_{k \geq j}\left\|\varphi_{k}-\varphi_{k+1}\right\|_{L^{p}}
$$


so that, by (A.8), we have

$$
\left\|E_{j}(\varphi)-E_{j-1}(\varphi)\right\|_{L^{p}} \leq 3 \sum_{k \geq j}\left\|\varphi_{k}-\varphi_{k-1}\right\|_{L^{p}} .
$$

Thus, by Hölder,

$$
\begin{aligned}
\left\|E_{j}(\varphi)-E_{j-1}(\varphi)\right\|_{L^{p}} & \leq 3 \sum_{k \geq j}(k-j+1)\left\|\varphi_{k}-\varphi_{k-1}\right\|_{L^{p}} \frac{1}{(k-j+1)} \\
& \leq 3\left(\sum_{k \geq j}(k-j+1)^{p}\left\|\varphi_{k}-\varphi_{k-1}\right\|_{L^{p}}^{p}\right)^{1 / p}\left(\sum_{k \geq j} \frac{1}{(k-j+1)^{p^{\prime}}}\right)^{1 / p^{\prime}}
\end{aligned}
$$

and therefore

$$
\left\|E_{j}(\varphi)-E_{j-1}(\varphi)\right\|_{L^{p}}^{p} \leq C \sum_{k \geq j}(k-j+1)^{p}\left\|\varphi_{k}-\varphi_{k-1}\right\|_{L^{p}}^{p}
$$

Consequently

$$
\begin{gathered}
\sum_{j \geq 1} 2^{s p j}\left\|E_{j}(\varphi)-E_{j-1}(\varphi)\right\|_{L^{p}}^{p} \leq C \sum_{j \geq 1} \sum_{k \geq j} 2^{s p j}(k-j+1)^{p}\left\|\varphi_{k}-\varphi_{k-1}\right\|_{L^{p}}^{p} \\
=C \sum_{k \geq 1} 2^{s p k}\left\|\varphi_{k}-\varphi_{k-1}\right\|_{L^{p}}^{p} a_{k}
\end{gathered}
$$

where

$$
\begin{aligned}
a_{k} & =\sum_{1 \leq j \leq k} 2^{s p(j-k)}(k-j+1)^{p} \\
& =2^{s p} \sum_{1 \leq \ell \leq k} \frac{\ell^{p}}{2^{s p \ell}} \leq a_{\infty}=2^{s p} \sum_{\ell=1}^{\infty} \frac{\ell^{p}}{2^{s p \ell}} .
\end{aligned}
$$

We deduce from (A.11) and Theorem A.1 that $\varphi \in W^{s, p}$ and

$$
\|\varphi\|_{W^{s, p}}^{p} \leq C \sum_{j \geq 1} 2^{s p j}\left\|\varphi_{j}-\varphi_{j-1}\right\|_{L^{p}}^{p} .
$$

Proof of Theorem A.1. Set

$$
\begin{aligned}
X & =\|f\|_{W^{s, p}}^{p} \\
Y & =\sum_{j \geq 1} 2^{s p j}\left\|E_{j}(f)-E_{j-1}(f)\right\|_{L^{p}}^{p} \\
Z & =\sum_{j \geq 0} 2^{s p j}\left\|f-E_{j}(f)\right\|_{L^{p}}^{p} .
\end{aligned}
$$


We will prove that $Y \sim Z$ and $Z \leq C X$ without assuming $s p<1$. That condition enters only to prove that $X \leq C Y$.

Step 1: $Y \leq Z$

Proof. We have, since $E_{j-1}(f) \in \mathcal{E}_{j-1} \subset \mathcal{E}_{j}$,

$$
E_{j}\left(E_{j-1}(f)\right)=E_{j-1}(f)
$$

and thus

$$
\left|E_{j}(f)-E_{j-1}(f)\right|=\left|E_{j}(f)-E_{j}\left(E_{j-1}(f)\right)\right| .
$$

Therefore

$$
\left\|E_{j}(f)-E_{j-1}(f)\right\|_{L^{p}} \leq\left\|f-E_{j-1}(f)\right\|_{L^{p}}
$$

and the estimate $Y \leq Z$ follows.

Step 2: $Z \leq C Y$. Here the condition $s p<1$ is not used; it suffices to have $s>0$.

Proof. Set $\varphi_{j}=E_{j}(f)$; as in the proof of Corollary A.1 we obtain

$$
\left\|f-\varphi_{j}\right\|_{L^{p}} \leq \sum_{k \geq j+1}\left\|\varphi_{k}-\varphi_{k-1}\right\|_{L^{p}}
$$

and, by Hölder,

$$
\left\|f-\varphi_{j}\right\|_{L^{p}} \leq\left(\sum_{k \geq j+1}(k-j)^{p}\left\|\varphi_{k}-\varphi_{k-1}\right\|_{L^{p}}^{p}\right)^{1 / p}\left(\sum_{k \geq j+1} \frac{1}{(k-j)^{p^{\prime}}}\right)^{1 / p^{\prime}}
$$

Thus

$$
\left\|f-\varphi_{j}\right\|_{L^{p}}^{p} \leq C \sum_{k \geq j+1}(k-j)^{p}\left\|\varphi_{k}-\varphi_{k-1}\right\|_{L^{p}}^{p}
$$

and consequently

$$
\begin{aligned}
\sum_{j \geq 0} 2^{s p j}\left\|f-\varphi_{j}\right\|_{L^{p}}^{p} & \leq C \sum_{j \geq 0} \sum_{k \geq j+1} 2^{s p j}(k-j)^{p}\left\|\varphi_{k}-\varphi_{k-1}\right\|_{L^{p}}^{p} \\
& =C \sum_{k \geq 1} 2^{s p k} a_{k}\left\|\varphi_{k}-\varphi_{k-1}\right\|_{L^{p}}^{p}
\end{aligned}
$$

where

$$
a_{k}=\sum_{0 \leq j \leq k-1} 2^{s p(j-k)}(k-j)^{p} \leq a_{\infty}=\sum_{\ell=1}^{\infty} \frac{\ell^{p}}{2^{s p \ell}}<\infty .
$$

Hence

$$
Z \leq C a_{\infty} Y
$$


Step 3: $Z \leq C X$. Here, again, the condition $s p<1$ is not used.

Proof. Recall that $Q_{j}(x)$ is the cube in the partition $\mathcal{P}_{j}$ containing the point $x$. Write

$$
\begin{aligned}
f(x)-E_{j}(f)(x) & =f(x)-\oint_{Q_{j}(x)} f(y) d y=\oint_{Q_{j}(x)}(f(x)-f(y)) d y \\
& =2^{n j} \int_{Q_{j}(x)}(f(x)-f(y)) d y
\end{aligned}
$$

and thus, by Hölder,

$$
\left|f(x)-E_{j}(f)(x)\right|^{p} \leq 2^{n j} \int_{Q_{j}(x)}|f(x)-f(y)|^{p} d y .
$$

Therefore

$$
\left\|f-E_{j}(f)\right\|_{L^{p}}^{p} \leq 2^{n j} \int_{\Omega} d x \int_{Q_{j}(x)}|f(x)-f(y)|^{p} d y
$$

so that

$$
\begin{aligned}
Z & =\sum_{j \geq 0} 2^{s p j}\left\|f-E_{j}(f)\right\|_{L^{p}}^{p} \leq \sum_{j \geq 0} 2^{(n+s p) j} \int_{\Omega} d x \int_{Q_{j}(x)}|f(x)-f(y)|^{p} d y \\
& =\int_{\Omega} \int_{\Omega} \frac{|f(x)-f(y)|^{p}}{|x-y|^{n+s p}} a(x, y) d x d y
\end{aligned}
$$

where

$$
a(x, y)=|x-y|^{n+s p} \sum_{j \geq 0} 2^{(n+s p) j} \mathbf{1}_{Q_{j}(x)}(y)
$$

and 1 denotes the characteristic function. Clearly

$$
a(x, y) \leq(4 n)^{(n+s p) / 2} \quad \forall x, y \in \Omega
$$

and the conclusion follows.

Step 4: $X \leq C Y$ when $s p<1$.

Proof. For $h \in \mathbb{R}^{n}$ set

$$
\left(\delta_{h} f\right)(x)=f(x+h)-f(x), x \in \Omega_{h}=\Omega \cap(\Omega-h) .
$$

A quantity equivalent to $X$ is

$$
X^{\prime}=\int_{|h|<1} \frac{d h}{|h|^{n+s p}} \int_{\Omega_{h}}\left|\left(\delta_{h} f\right)(x)\right|^{p} d x .
$$

We will use the following two lemmas 
Lemma A.1. We have, with some constant $C$ (depending only on $p, \alpha$ and $\beta$ ), for all $h \in \mathbb{R}^{n}$ and all $j \geq 1$

$$
\left\|\delta_{h} f\right\|_{L^{p}\left(\Omega_{h}\right)}^{p} \leq C\left(\sum_{k=1}^{j} 2^{\alpha(j-k) p}\left\|\delta_{h}\left(f_{k}-f_{k-1}\right)\right\|_{L^{p}\left(\Omega_{h}\right)}^{p}+\sum_{k=j+1}^{\infty} 2^{\beta(k-j) p}\left\|f_{k}-f_{k-1}\right\|_{L^{p}(\Omega)}^{p}\right),
$$

where $\alpha>0$ and $\beta>0$ will be chosen later.

Proof. As above, write

$$
f=f_{0}+\sum_{k \geq 1}\left(f_{k}-f_{k-1}\right)
$$

and thus

$$
\delta_{h} f=\sum_{k \geq 1} \delta_{h}\left(f_{k}-f_{k-1}\right),
$$

so that

$$
\left\|\delta_{h} f\right\|_{L^{p}\left(\Omega_{h}\right)} \leq \sum_{k=1}^{j}\left\|\delta_{h}\left(f_{k}-f_{k-1}\right)\right\|_{L^{p}\left(\Omega_{h}\right)}+2 \sum_{k=j+1}^{\infty}\left\|f_{k}-f_{k-1}\right\|_{L^{p}(\Omega)},
$$

and the conclusion follows from Hölder's inequality.

Lemma A.2. We have, for all $h \in \mathbb{R}^{n}$ and all $\psi \in \mathcal{E}_{k}, k \geq 1$,

$$
\left\|\delta_{h} \psi\right\|_{L^{p}\left(\Omega_{h}\right)}^{p} \leq C|h| 2^{k}\|\psi\|_{L^{p}(\Omega)}^{p}
$$

where $C$ depends only on $p$ and $n$.

Proof. Write

$$
\psi=\sum_{Q \in \mathcal{P}_{k}} a_{Q} \mathbf{1}_{Q}
$$

and thus

$$
\delta_{h} \psi=\sum_{Q} a_{Q}\left(\delta_{h} \mathbf{1}_{Q}\right)
$$

Therefore, by Hölder

$$
\left|\delta_{h} \psi\right|^{p} \leq\left(\sum_{Q}\left|a_{Q}\right|^{p}\left|\delta_{h} \mathbf{1}_{Q}\right|\right)\left(\sum_{Q}\left|\delta_{h} \mathbf{1}_{Q}\right|\right)^{p-1} .
$$

But

$$
\sum_{Q}\left|\delta_{h} \mathbf{1}_{Q}\right| \leq 2
$$


and thus

$$
\int_{\Omega_{h}}\left|\delta_{h} \psi\right|^{p} \leq C \sum_{Q}\left|a_{Q}\right|^{p} \int_{\Omega_{h}}\left|\delta_{h} \mathbf{1}_{Q}\right|
$$

On the other hand

$$
\int_{\Omega_{h}}\left|\delta_{h} \mathbf{1}_{Q}\right| \leq|Q \backslash(Q-h)|+|(Q-h) \backslash Q| \leq C \frac{|h|}{2^{(n-1) k}}
$$

and

$$
\|\psi\|_{L^{p}(\Omega)}^{p}=\frac{1}{2^{n k}} \sum_{Q}\left|a_{Q}\right|^{p}
$$

Combining (A.15), (A.16) and (A.17) yields (A.14).

Proof of Step 4 completed. In view of (A.13) we have

$$
X \leq C \sum_{j=1}^{\infty} \int_{\frac{1}{2 j}<|h|<\frac{1}{2^{j}-1}} \frac{d h}{|h|^{n+s p}} \int_{\Omega_{h}}\left|\left(\delta_{h} f\right)(x)\right|^{p} d x .
$$

Combining this with Lemma A.1 we find

$$
X \leq C\left(I_{1}+I_{2}\right)
$$

where

$$
I_{1}=\sum_{j=1}^{\infty} \int_{\frac{1}{2^{j}}<|h|<\frac{1}{2^{j}-1}} 2^{(n+s p) j} \sum_{k=1}^{j} 2^{\alpha(j-k) p}\left\|\delta_{h}\left(f_{k}-f_{k-1}\right)\right\|_{L^{p}\left(\Omega_{h}\right)}^{p} d h
$$

and

$$
I_{2}=\sum_{j=1}^{\infty} \int_{\frac{1}{2^{j}}<|h|<\frac{1}{2^{j}-1}} 2^{(n+s p) j} \sum_{k=j+1}^{\infty} 2^{\beta(k-j) p}\left\|f_{k}-f_{k-1}\right\|_{L^{p}(\Omega)}^{p} d h .
$$

The estimate for $I_{2}$ is very simple since

$$
\begin{aligned}
I_{2} & \leq C \sum_{j=1}^{\infty} \sum_{k=j+1}^{\infty} 2^{s p j} 2^{\beta(k-j) p}\left\|f_{k}-f_{k-1}\right\|_{L^{p}}^{p} \\
& =C \sum_{k=2}^{\infty} 2^{s p k} b_{k}\left\|f_{k}-f_{k-1}\right\|_{L^{p}}^{p}
\end{aligned}
$$


where

$$
b_{k}=\sum_{j=1}^{k-1} 2^{s p(j-k)} 2^{\beta(k-j) p} \leq b=\sum_{\ell=1}^{\infty} 2^{(\beta-s) \ell p}<\infty
$$

provided we choose $0<\beta<s$. Therefore $I_{2} \leq C Y$.

To estimate $I_{1}$ we apply Lemma A.2 with $\psi=\left(f_{k}-f_{k-1}\right)$. Inserting (A.14) in (A.18) we obtain

$$
\begin{aligned}
I_{1} & \leq C \sum_{j=1}^{\infty} 2^{s p j} \sum_{k=1}^{j} 2^{(k-j)} 2^{\alpha(j-k) p}\left\|f_{k}-f_{k-1}\right\|_{L^{p}}^{p} \\
& =C c \sum_{k=1}^{\infty} 2^{s p k}\left\|f_{k}-f_{k-1}\right\|_{L^{p}}^{p}
\end{aligned}
$$

with

$$
c=\sum_{\ell=0}^{\infty} 2^{(s p-1+\alpha p) \ell}<\infty
$$

provided we choose $0<\alpha<(1-s p) / p$ (this is the only place where we use the assumption $s p<1)$. Thus we have proved that $I_{1} \leq C Y$ and the proof of Step 4 is complete.

Returning to Theorem A.1 it is a natural question to ask how the norm-equivalence deteriorates when $s p \rightarrow 1$. It was already observed that the inequality

$$
\sum_{j \geq 1} 2^{s p j}\left\|\Delta_{j} f\right\|_{L^{p}}^{p} \leq C\|f\|_{W^{s, p}}^{p}
$$

where $\Delta_{j} f=E_{j}(f)-E_{j-1}(f)$, is independent of the assumption $s p<1$. Concerning the other direction, one has the following more precise result when $s p$ is close to 1.

Proposition A.1. Assume $s p<1$. Then

$$
\|f\|_{W^{s, p}} \leq \frac{C}{s(1-s p)}\left(\sum_{j \geq 1} 2^{s p j}\left\|\Delta_{j} f\right\|_{L^{p}}^{p}\right)^{1 / p}
$$

where $C$ is an absolute constant.

Proof. Following the proof of Step 4 with

$$
\alpha=(1-s p) / 2 p \quad \text { and } \beta=s / 2
$$

and using the fact that

$$
\sum_{\ell=1}^{\infty} 2^{-a \ell} \leq \int_{0}^{\infty} \frac{d x}{2^{a x}}=C / a
$$


we obtain

$$
X \leq\left(1+\frac{C}{\alpha p^{\prime}}+\frac{C}{\beta p^{\prime}}\right)^{p-1}\left(I_{1}+I_{2}\right)
$$

and then

$$
\begin{aligned}
& I_{2} \leq C\left(1+\frac{1}{s p}\right) Y \\
& I_{1} \leq \frac{C}{1-s p} Y .
\end{aligned}
$$

Combining these inequalities yields (A.20).

In particular, with $p=2$, we find

Corollary A.2. For $1 / 4<s<1 / 2$ we have

$$
\|f\|_{H^{s}} \leq C(1-2 s)^{-1}\left(\sum_{j \geq 1} 4^{s j}\left\|\Delta_{j} f\right\|_{L^{2}}^{2}\right)^{1 / 2}
$$

where $C$ is an absolute constant.

The dependence in $(1-2 s)^{-1}$ for $s \rightarrow 1 / 2$ in Corollary A.2 is optimal as can be seen from the following example.

Lemma A.3. Let $0<s<\frac{1}{2}$. Let $\Omega=(-1,1)$ equipped with standard dyadic partition $\left\{\mathcal{P}_{j}\right\}$ and

$$
f=\left(\log \frac{1}{x}\right) \chi_{[0<x<1]}
$$

Then

$$
\begin{aligned}
& \text { (i) } \quad\|f\|_{H^{s}} \gtrsim(1-2 s)^{-3 / 2} \\
& \text { (ii) } \quad\left(\sum_{j \geq 1} 4^{j s}\left\|\Delta_{j} f\right\|_{L^{2}}^{2}\right)^{1 / 2} \sim(1-2 s)^{-1 / 2} .
\end{aligned}
$$

Proof.

$(i)$

$$
\begin{aligned}
\|f\|_{H^{s}}^{2}=\iint \frac{|f(x+h)-f(x)|^{2}}{|h|^{1+2 s}} d x d h & \geq \iint_{x<0<x+h} h^{-(1+2 s)}\left(\log \frac{1}{x+h}\right)^{2} d x d h \\
& \geq \sum_{j} 4^{j s} \int_{-2^{-j+1}}^{-2^{-j}}\left(\log \frac{1}{x}\right)^{2} d x \\
& \sim \sum_{j} j^{2} 2^{-j(1-2 s)} \\
& \sim(1-2 s)^{-3} .
\end{aligned}
$$


(ii) We need to evaluate the increments $\Delta_{j} f$. Let $I \in \mathcal{P}_{j-1}$,

$$
I=\left[a, a+2^{-(j-1)}\right] \subset[0,1] .
$$

Thus the value of $\left|\Delta_{j} f\right|$ on $I$ is

$$
2^{j}\left|\int_{a}^{a+2^{-j}} f-\int_{a+2^{-j}}^{a+2^{-j+1}} f\right|=2^{j}\left|F\left(a+2^{-j+1}\right)+F(a)-2 F\left(a+2^{-j}\right)\right|
$$

where

$$
F(x)=x \log \frac{1}{x}+x
$$

For $a=0$,

$$
(A .21)=2^{j}\left|F\left(2^{-j+1}\right)-2 F\left(2^{-j}\right)\right|=2^{j}\left|2^{-j+1}(j-1)-2^{-j+1} j\right|=2 .
$$

For $a=r 2^{-(j-1)}, r \geq 1$

$$
(A .21) \lesssim 2^{j} 4^{-j}\left\|F^{\prime \prime}\right\|_{L^{\infty}(I)}=2^{-j}\left\|\frac{1}{x}\right\|_{L^{\infty}(I)} \sim \frac{1}{r} .
$$

It follows in particular from (A.22), (A.23) that

$$
\begin{gathered}
\left\|\Delta_{j} f\right\|_{2}^{2} \leq C 2^{-j} \sum_{r \geq 1} r^{-2}=C 2^{-j} \\
\sum 4^{j s}\left\|\Delta_{j} f\right\|_{2}^{2} \leq C \sum 2^{-j(1-2 s)} \sim(1-2 s)^{-1} .
\end{gathered}
$$

\section{APPENDIX B}

\section{Functions in $\mathrm{W}^{\mathrm{s}, \mathrm{p}}(\Omega ; \mathbb{Z})$ are constant when $\mathrm{sp} \geq \mathbf{1}$.}

A continuous function on a connected space with values into $\mathbb{Z}$ must be constant. Functions in the Sobolev space $W^{s, p}$ with $s p \geq 1$ have the same property although they need not be continuous. More precisely we have

Theorem B.1. Assume $\Omega$ is a connected open set in $\mathbb{R}^{n}, n \geq 1$. Let $0<s<\infty$ and $1<p<\infty$ be such that

$$
s p \geq 1,
$$

including $s=1$ and $p=1$. Then any function $f \in W^{s, p}(\Omega ; \mathbb{Z})$ must be constant.

Remark B.1. Hardt, Kinderlehrer and Lin [1] have stated the same conclusion when $s=1 / 2$ and $p=2$ with a sketch of proof. Bethuel and Demengel [1] have also obtained the same result when $s p>1$ and the proof we present follows their argument with an additional ingredient to cover the case $s p=1$. 
Proof. It is convenient to split the proof into two steps:

\section{Step 1: the case $\mathbf{n}=1$.}

If $s p>1$, the conclusion is obvious since $f$ is continuous by the Sobolev imbedding theorem. If $s p=1$, a borderline for the Sobolev imbedding, $f$ need not be continuous, but $f$ is VMO (see e.g. Brezis and Nirenberg [1], Section I.2). Therefore, the essential range of $f$ is connected (see Brezis and Nirenberg [1], Section I.5) and thus $f$ is constant. For the convenience of the reader we reproduce the argument. Set

$$
f_{\varepsilon}(x)=\oint_{B_{\varepsilon}(x)} f(y) d y
$$

and note that

$$
\operatorname{dist}\left(f_{\varepsilon}(x), \mathbb{Z}\right) \leq \oint_{B_{\varepsilon}(x)}\left|f(y)-f_{\varepsilon}(x)\right| d y \rightarrow 0
$$

uniformly in $x$ as $\varepsilon \rightarrow 0$ (since $f \in \mathrm{VMO}$ ). On the other hand $f_{\varepsilon}(\Omega)$ is connected and consequently there is some integer $k_{\varepsilon} \in \mathbb{Z}$ such that

$$
\left\|f_{\varepsilon}-k_{\varepsilon}\right\|_{L^{\infty}} \rightarrow 0 \quad \text { as } \varepsilon \rightarrow 0
$$

It follows that $k_{\varepsilon} \rightarrow k$ as $\varepsilon \rightarrow 0$ with $k \in \mathbb{Z}$ and $f=k$ a.e. on $\Omega$.

\section{Step 2: the case $n \geq 2$.}

It suffices to prove that $f$ is locally constant a.e. and thus we may assume, without loss of generality, that $\Omega=(0,1)^{n}$. For a.e. $x^{\prime}=\left(x_{1}, \ldots, x_{i-1}, x_{i+1}, \ldots x_{n}\right)$ in $(0,1)^{n-1}$ the function

$$
t \mapsto \psi(t)=f\left(x_{1}, \ldots x_{i-1}, t, x_{i+1}, \ldots x_{n}\right)
$$

belongs to $W^{s, p}(0,1)$. This is a consequence of the fact that an equivalent norm for $W^{s, p}\left(\mathbb{R}^{n}\right)(0<s<1)$ is given by

$$
\|\| f \mid\left\|^{p}=\right\| f \|_{L^{p}}^{p}+\sum_{i=1}^{n} \int_{0}^{1} \int_{\mathbb{R}^{n}} \frac{\left|f\left(x+t e_{i}\right)-f(x)\right|^{p}}{t^{1+s p}} d x d t
$$

where $\left(e_{i}\right)$ denotes the canonical basis of $\mathbb{R}^{n}$ (see e.g. Adams [1], p.208-214). Applying Step 1 we know that for a.e. $x^{\prime} \in(0,1)^{n-1}$ the function $\psi$ is constant. To complete Step 2 we rely on the following simple measure theoretical lemma (see e.g. Lemma 2 in Brezis, Li, Mironescu and Nirenberg [1]) 
Lemma B.1. Let $\Omega=(0,1)^{n}$ and let $f$ be a measurable function on $\Omega$ such that for each $1 \leq i \leq n$ and for a.e. $x^{\prime}=\left(x_{1}, \ldots, x_{i-1}, x_{i+1}, \ldots x_{n}\right)$ in $(0,1)^{n-1}$ the function $\psi$ defined in (B.2) is constant a.e. on $(0,1)$. Then $f$ is constant a.e. on $\Omega$.

Remark B.2. Assumption (B.1) cannot be weakened. Indeed, the characteristic function of any smooth domain $\omega$ compactly contained in $\Omega$ belongs to $W^{s, p}$ for any $s, p$ with $s p<1$.

Remark B.3. The conclusion of Theorem B.1 is still valid if $f: \Omega \rightarrow \mathbb{Z}$ is a sum of functions in different Sobolev space, i.e., $f=\sum_{i=1}^{k} f_{i}$ with $f_{i} \in W^{s_{i}, p_{i}}(\Omega ; \mathbb{R})$ and $s_{i} p_{i} \geq 1$ for all $i$. The proof is identical to the one we have presented above. In particular the conclusion holds if $f \in H^{1 / 2}+W^{1,1}$; this fact will be used in our forthcoming paper Bourgain, Brezis and Mironescu [1].

\section{APPENDIX C \\ Composition in fractional Sobolev spaces}

We investigate here the question whether $\Phi \circ v$ belongs to $W^{s, p}(\Omega)$ when $v$ belongs to $W^{s, p}(\Omega)$ and $\Phi$ is smooth. For simplicity we consider only the case $\Omega=\mathbb{R}^{n}$. Of course, here, we also assume that $\Phi(0)=0$. The case of a domain can be treated by extending the functions to $\mathbb{R}^{n}$.

Lemma C.1. Let $0<s<\infty$ and $1<p<\infty$. Assume

$$
v \in W^{s, p}(\Omega) \cap L^{\infty}(\Omega) .
$$

Then

$$
\Phi \circ v \in W^{s, p}(\Omega) .
$$

Proof. When $s$ is an integer the conclusion is easy via the Gagliardo-Nirenberg inequality. For example, when $s=2$

$$
D^{2}(\Phi \circ v)=\Phi^{\prime}(v) D^{2} v+\Phi^{\prime \prime}(v)(D v)^{2} \in L^{p}
$$

since $W^{2, p} \cap L^{\infty} \subset W^{1,2 p}$ by the Gagliardo-Nirenberg inequality. A similar argument holds for higher order derivatives.

We now turn to the case where $s$ is fractional. The conclusion is obvious when $0<s<1$. Suppose now that $1<s<2$. One has to show that

$$
D(\Phi \circ v)=\Phi^{\prime}(v) D v \in W^{s-1, p} .
$$

This would require a lemma about products which eludes us.

Instead of this strategy one relies on a characterization of $W^{s, p}$ via finite differences. Set

$$
\left(\delta_{h} u\right)(x)=u(x+h)-u(x),
$$


so that

$$
\left(\delta_{h}^{2} u\right)(x)=u(x+2 h)-2 u(x+h)+u(x) .
$$

Then

$$
u \in W^{s, p} \Leftrightarrow \iint \frac{\left|\delta_{h}^{2} u(x)\right|^{p}}{|h|^{n+s p}} d h d x<\infty
$$

(see Triebel [2], p.110).

The key observation is that $\delta_{h}^{2}(\Phi \circ v)$ can be estimated in terms of $\delta_{h}^{2} v$ and $\delta_{h} v$. This is the purpose of our next computation.

Set

$$
\begin{aligned}
X & =v(x+2 h) \\
Y & =v(x+h) \\
Z & =v(x) .
\end{aligned}
$$

Then

$$
\Phi(X)-\Phi(Y)=\Phi^{\prime}(Y)(X-Y)+O\left(|X-Y|^{2}\right)
$$

and

$$
\Phi(Z)-\Phi(Y)=\Phi^{\prime}(Y)(Z-Y)+O\left(|Z-Y|^{2}\right)
$$

Since

$$
\delta_{h}^{2}(\Phi \circ v)(x)=(\Phi(X)-\Phi(Y))+(\Phi(Z)-\Phi(Y))
$$

one finds

$$
\left|\delta_{h}^{2}(\Phi \circ v)(x)\right| \leq C\left(\left|\delta_{h}^{2} v(x)\right|+\left|\delta_{h} v(x+h)\right|^{2}+\left|\delta_{h} v(x)\right|^{2}\right) .
$$

Consequently

$$
\iint \frac{\left|\delta_{h}^{2}(\Phi \circ v)(x)\right|^{p}}{|h|^{n+s p}} \leq C \iint \frac{\left|\delta_{h}^{2} v(x)\right|^{p}}{|h|^{n+s p}}+C \iint \frac{\left|\delta_{h} v(x)\right|^{2 p}}{|h|^{n+s p}}
$$

The first term on the righthand side of (C.7) is finite since $v \in W^{s, p}$ and for the second term we observe that

$$
\iint \frac{\left|\delta_{h} v(x)\right|^{2 p}}{|h|^{n+s p}}=\|v\|_{W^{\frac{s}{2}, 2 p}}^{2 p} \leq C\|v\|_{L^{\infty}}^{p}\|v\|_{W^{s, p}}^{p}
$$


by the Gagliardo-Nirenberg inequality (see Lemma D.1). Hence we have proved that $\Phi \circ v \in W^{s, p}$. The same argument extends to a general $s>2, s$ non integer (see e.g. Escobedo [1]).

\section{APPENDIX D}

\section{Gagliardo-Nirenberg inequalities and products in fractional Sobolev spaces}

We establish here some Gagliardo- Nirenberg type inequalities used in the paper. We also present a proof of Lemma 2 concerning products in fractional Sobolev spaces. These results are presumably known to the experts. For simplicity we work on $\mathbb{R}^{n}$; the case of a domain can be treated by extending the functions to $\mathbb{R}^{n}$.

Lemma D.1. Let $0<s<\infty, 1<p<\infty$. Assume

$$
u \in W^{s, p}\left(\mathbb{R}^{n}\right) \cap L^{\infty}\left(\mathbb{R}^{n}\right) .
$$

Then

$$
u \in W^{r, q}, \quad \forall r \in(0, s) \quad \text { with } q=\frac{s p}{r},
$$

and

$$
\left|\left\|u \left|\| _ { W ^ { r , q } } \leq C \| u \left\|_ { L ^ { \infty } } ^ { 1 - ( r / s ) } \left|\|u \mid\|_{W^{s, p}}^{r / s},\right.\right.\right.\right.\right.
$$

provided that either (i) both $r, s$ are non integers or (ii) $r$ is an integer.

Here, we use the following semi-norm on $W^{s, p}$ (see e.g. Triebel [2]):

$$
\left|\|u \mid\|_{W^{s, p}}= \begin{cases}\left\|D^{s} u\right\|_{L^{p}}, & \text { if } s \text { is an integer } \\ \left(\iint \frac{\left|\delta_{h}^{M} u(x)\right|^{p}}{|h|^{n+s p}} d x d h\right)^{1 / p} & \text { if } s \text { is not an integer }\end{cases}\right.
$$

(as usual, $M>s$ is any integer).

Proof of Lemma D.1. It is convenient to observe that, for every $s \in(0, \infty)$ and every $p \in(1, \infty)$

$$
\left|\left\|u \left|\left\|_ { W ^ { s , p } ( \mathbb { R } ^ { n } ) } ^ { p } \sim \int _ { S ^ { n - 1 } } d \sigma \int _ { y \cdot \sigma = 0 } \left|\|u(t \sigma+y) \mid\|_{W^{s, p}(\mathbb{R})}^{p} d y .\right.\right.\right.\right.\right.
$$

(When $s$ is not an integer, (D.3) is clear. When $s$ is an integer, (D.3) follows from the fact that the function

$$
A \mapsto\left(\int_{S^{n-1}}|A(\sigma, \sigma, \ldots, \sigma)|^{p} d \sigma\right)^{\frac{1}{p}}
$$


is a norm on the space of $s$-linear symmetric forms on $\mathbb{R}^{n}$.) Using (D.3) one sees that the proof of (D.2) reduces to the one-dimensional case.

Also, note that the desired inequality (D.2) is clear when both $s$ and $r$ are not integers. Indeed, in this case, we have, for $M>s$ (and hence $M>r$ )

$$
\begin{aligned}
\|\| u \mid \|_{W^{r, q}}^{q} & =\iint \frac{\left|\delta_{h}^{M} u(x)\right|^{q}}{|h|^{n+r q}} d x d h \leq\left\|\delta_{h}^{M} u\right\|_{L^{\infty}}^{q-p} \iint \frac{\left|\delta_{h}^{M} u(x)\right|^{p}}{|h|^{n+r q}} d x d h \\
& \leq C\|u\|_{L^{\infty}}^{q-p}\left|\|u \mid\|_{W^{s, p}}^{p} .\right.
\end{aligned}
$$

Therefore, it suffices to establish (D.2) for $n=1$ and $s \geq 1$. We follow the proof of Nirenberg [1]. By the Sobolev imbedding theorem, we have (since $s p>1$ ),

$$
W^{s, p}([0,1]) \subset W^{r, q}([0,1]) .
$$

Hence

$$
\|\| u \mid \|_{W^{r, q}([0,1])} \leq C\left(\|u\|_{L^{p}([0,1])}+\left|\|u \mid\|_{W^{s, p}([0,1])}\right), u \in W^{s, p}([0,1]) .\right.
$$

It then follows that

$$
\left|\|u \mid\|_{W^{r, q}([0,1])} \leq C\left(\|u\|_{L^{\infty}([0,1])}+\left|\|u \mid\|_{W^{s, p}([0,1])}\right), u \in W^{s, p}([0,1]) .\right.\right.
$$

By scaling, we find

$$
\begin{aligned}
\left|\|u \mid\|_{W^{r, q}([0, \ell])}^{q}\right. & \leq C\left(\ell^{1-s p}\|u\|_{L^{\infty}([0, \ell])}^{q}+\ell^{\left(\frac{s}{r}-1\right)(s p-1)}\left|\|u \mid\|_{W^{s, p}([0, \ell])}^{q}\right)\right. \\
& =C(A(\ell)+B(\ell)), u \in W^{s, p}([0, \ell])
\end{aligned}
$$

It clearly suffices to prove (D.2) in $[0, \infty)$ and we may assume that $\|u\|_{W^{s, p}}=1$. Fix some $\varepsilon>0$. We construct inductively a sequence of disjoint intervals $I_{1}, I_{2}, \ldots$ such that

$$
[0,+\infty)=I_{1} \cup I_{2} \cup \cdots
$$

as follows:

We compare $A(\varepsilon)$ and $B(\varepsilon)$. If $B(\varepsilon) \geq A(\varepsilon)$, then we take $I_{1}=[0, \varepsilon)$ and next construct $I_{2}$. Otherwise, note that $\lim _{\ell \rightarrow \infty} A(\ell)=0, \lim _{\ell \rightarrow \infty} B(\ell)=\infty$ (unless $u \equiv 0$, which is not the case). Hence there is some $\varepsilon<\ell<\infty$ such that $A(\ell)=B(\ell)$. It then follows that

$$
\|\| u\left|\left\|_{W^{r, q}([0, \ell])}^{q} \leq C\right\| u\left\|_{L^{\infty}([0, \ell])}^{q-p} \mid\right\| u \|_{W^{s, p}([0, \ell])}^{p} .\right.
$$

In this case we take $I_{1}=[0, \ell)$. We next start the above procedure from the endpoint of $I_{1}$. Since at each step we have $\left|I_{j}\right| \geq \varepsilon$, we clearly cover in this way $[0, \infty)$ with a sequence 
of intervals. Denote the first type of intervals by $I_{j}$ and the second type by $K_{j}$. Using the assumption that $r$ is an integer we have

$$
\begin{aligned}
\|\| u \mid \|_{W^{r, q}([0, \infty))}^{q} & =\sum_{I_{j}}\left|\|u \mid\|_{W^{r, q}\left(I_{j}\right)}^{q}+\sum_{K_{j}} \cdots\right. \\
& \leq C \varepsilon^{\left(\frac{s}{r}-1\right)(s p-1)} \sum_{I_{j}} \mid\|u\|_{W^{s, p}\left(I_{j}\right)}^{q} \\
& +C\|u\|_{L^{\infty}(\mathbb{R})}^{q-p} \sum_{K_{j}}\left|\|u \mid\|_{W^{s, p}\left(K_{j}\right)}^{p} .\right.
\end{aligned}
$$

Note that, since $q>p$, we have

$$
\sum_{I_{j}}\left|\left\|u \left|\left\|_{W^{s, p}\left(I_{j}\right)}^{p} \leq 1 \Rightarrow \sum_{I_{j}}\left|\|u \mid\|_{W^{s, p}\left(I_{j}\right)}^{q} \leq 1 .\right.\right.\right.\right.\right.
$$

Hence

$$
\left|\left\|u \left|\| _ { W ^ { r , q } ( [ 0 , \infty ] ) } ^ { q } \leq C \varepsilon ^ { ( \frac { s } { r } - 1 ( s p - 1 ) } + C \| u \left\|_ { L ^ { \infty } ( \mathbb { R } ) } ^ { q - p } \left|\|u \mid\|_{W^{s, p}(\mathbb{R})}^{p} .\right.\right.\right.\right.\right.
$$

We conclude by letting $\varepsilon \rightarrow 0$ in (D.7) (the constants $C$ are independent of $\varepsilon$ ).

Remark D.1. The conclusion of Lemma D.1 fails when $s=1$ and $p=1$. For example $W^{1,1}(\mathbb{R}) \cap L^{\infty}(\mathbb{R})$ is not contained in $W^{1 / 2,2}(\mathbb{R})$ - because this would imply the inequality $\|u\|_{W^{1 / 2,2}} \leq C\|u\|_{W^{1,1}}$ which is clearly wrong (use for example the sequence in Remark 3 ).

Remark D.2. In the general case (no restrictions on $r$ and $s$ ), the conclusions of Lemma D.1 are still true (the remaining case, i.e., $s$ integer and $r$ non integer, is treated in $\mathrm{T}$. Runst [1], Lemma 5.2.1).

We next prove a regularity result for products in Sobolev spaces.

Lemma D.2. Let $n \geq 1,1<s<\infty, 1<p<\infty$. Let $u, v \in W^{s, p}\left(\mathbb{R}^{n}\right) \cap L^{\infty}\left(\mathbb{R}^{n}\right)$. Then

$$
u D v \in W^{s-1, p}\left(\mathbb{R}^{n}\right)
$$

Proof of Lemma D.2. If $s$ is an integer, the conclusion follows easily from the GagliardoNirenberg inequality. We henceforth assume that $s$ is not an integer.

We use a Littlewood- Paley decomposition technique (see e.g. Bony [1], Alinhac and Gérard [1] or Chemin [1]). Let $\psi_{0} \in C_{0}^{\infty}\left(\mathbb{R}^{n}\right)$ be such that

$$
\psi_{0}(\xi)=1 \quad \text { if }|\xi| \leq 1 \quad \text { and } \psi_{o}(\xi)=0 \quad \text { if }|\xi| \geq 2
$$

Set

$$
\psi_{j}(\xi)=\psi_{0}\left(2^{-j} \xi\right)-\psi_{0}\left(2^{-j+1} \xi\right), j \geq 1 \quad \text { and } \varphi_{j}=\mathcal{F}^{-1}\left(\psi_{j}\right), j \geq 0
$$


For $f \in \mathcal{S}^{\prime}$, let $f_{j}=f * \varphi_{j}$, so that $f=\sum_{j \geq 0} f_{j}$ in $\mathcal{S}^{\prime}$.

We have $u D v=\sum\left(r_{j}+s_{j}\right)$, where

$$
r_{j}=u_{j} \sum_{k \leq j-1} D v_{k} \quad \text { and } s_{j}=D v_{j} \sum_{k \leq j} u_{k} .
$$

Since clearly

$$
\left\|\sum_{k \leq j} \varphi_{k}\right\|_{L^{1}} \leq C, \quad\left\|\sum_{k \leq j} D \varphi_{k}\right\|_{L^{1}} \leq C 2^{j}, \quad \forall j \geq 0
$$

we obtain

$$
\begin{gathered}
\left\|\sum_{k \leq j} v_{k}\right\|_{L^{q}} \leq C\|v\|_{L^{q}}, \quad \forall q, \\
\left\|\sum_{k \leq j} D v_{k}\right\|_{L^{q}} \leq C 2^{j}\|v\|_{L^{q}}, \quad \forall q,
\end{gathered}
$$

and the same inequalities hold for $u$. Therefore,

$$
\left\|r_{j}\right\|_{L^{p}}^{p} \leq C\left\|u_{j}\right\|_{L^{p}}^{p}\left\|\sum_{k \leq j-1} D v_{k}\right\|_{L^{\infty}}^{p} \leq C 2^{j p}\left\|u_{j}\right\|_{L^{p}}^{p}\|v\|_{L^{\infty}}^{p}
$$

On the other hand, $v_{j}=\sum_{k \leq j+2}\left(v_{j}\right)_{k}$, since, for $k \geq j+3$,

$$
\mathcal{F}\left(\left(v_{j}\right)_{k}\right)=\mathcal{F}(v) \psi_{j} \psi_{k}=0 .
$$

Therefore,

$$
\left\|D v_{j}\right\|_{L^{q}}=\left\|\sum_{k \leq j+2} D\left(v_{j}\right)_{k}\right\|_{L^{q}} \leq C 2^{j}\left\|v_{j}\right\|_{L^{q}}, \quad \forall q,
$$

by (D.9) applied to $v_{j}$. Consequently,

$$
\left\|s_{j}\right\|_{L^{p}}^{p} \leq C\|u\|_{L^{\infty}}^{p}\left\|D v_{j}\right\|_{L^{p}}^{p} \leq C 2^{j p}\left\|v_{j}\right\|_{L^{p}}^{p}\|u\|_{L^{\infty}}^{p} .
$$

We now recall two basic facts about $W^{\sigma, p}, \sigma>0, \sigma$ non integer, $1<p<\infty$. Let $f \in W^{\sigma, p}$ and let $f_{j}=f * \varphi_{j}$ as above. Then

$$
\|f\|_{W^{\sigma, p}}^{p} \sim \sum_{j \geq 0} 2^{\sigma j p}\left\|f_{j}\right\|_{L^{p}}^{p}
$$

(see e.g. Triebel [2], p. 46). 
Conversely, let $g_{j}$ be a sequence in $L^{p}$ such that $\operatorname{supp} \mathcal{F}\left(g_{j}\right) \subset B_{2^{j}}$. Then

$$
\left\|\sum_{j \geq 0} g_{j}\right\|_{W^{\sigma, p}}^{p} \leq C \sum_{j \geq 0} 2^{\sigma j p}\left\|g_{j}\right\|_{L^{p}}^{p}
$$

(see e.g. Chemin [1], p. 27). Using (D.10), (D.11) and (D.12) (with $\sigma=s$ ), we find

$$
\sum_{j \geq 0} 2^{(s-1) j p}\left\|r_{j}+s_{j}\right\|_{L^{p}}^{p} \leq C\left(\|u\|_{L^{\infty}}^{p}\|v\|_{W^{s, p}}^{p}+\|v\|_{L^{\infty}}^{p}\|u\|_{W^{s, p}}^{p}\right) .
$$

Since supp $\mathcal{F}\left(r_{j}+s_{j}\right) \subset B_{2^{j+3}}$, (D.13) (applied with $\sigma=s-1$ and $g_{j}=r_{j}+s_{j}$ ) combined with (D.14) yields that $u D v \in W^{s-1, p}$ and that

$$
\|u D v\|_{W^{s-1, p}} \leq C\left(\|u\|_{L^{\infty}}\|v\|_{W^{s, p}}+\|v\|_{L^{\infty}}\|u\|_{W^{s, p}}\right) .
$$

Remark D.3. As a consequence of Lemma D.2, we derive the well-known fact that $W^{s, p} \cap L^{\infty}$ is an algebra.

\section{APPENDIX E}

\section{Behaviour of the $H^{s}$-norm of lifting for $s \overrightarrow{<} \frac{1}{2}$. Proof of Theorem 4}

We return to the particular issue of lifting of an unimodular function $F$ in $H^{s}, s<\frac{1}{2}$. As we have pointed out in Section 5 the construction described in Appendix A of a lifting

$$
F=e^{i \varphi}, \varphi \in H^{s}
$$

does not lead to the optimal estimate of $\|\varphi\|_{H^{s}}$ when $s \rightarrow \frac{1}{2}$. Our aim is to prove

Theorem E.1. Let $Q$ be a cube of $\mathbb{R}^{d}, d \geq 1$. For every $F \in H^{s}\left(Q ; S^{1}\right)$ with $0<s<1 / 2$ one may construct a $\varphi \in H^{s}(Q ; \mathbb{R})$ satisfying $(E .1)$ and the (optimal) estimate

$$
\|\varphi\|_{H^{s}} \leq C(1-2 s)^{-1 / 2}\|F\|_{H^{s}}
$$

where $C$ is a constant independent of $F$ and independent of $s$ as $s \rightarrow 1 / 2$.

Proof. Given an unimodular $H^{s}$-function $F$ on a cube, say $Q=\left[0, \frac{1}{2}\right]^{d} \subset \mathbb{R}^{d}$, we may extend $F$ to a 1-periodic unimodular function in $H_{\text {loc }}^{s}\left(\mathbb{R}^{d}\right)$ by the usual procedure of reflections and periodic continuation. Hence, we may assume $F \in H^{s}\left(\mathbb{T}^{d} ; S^{1}\right)$, where $\mathbb{T}^{d}=d$-dim torus. This setting is particularly convenient to perform our translation averaging. On $\Omega=\mathbb{T}^{d}$, we fix again a system $\left\{\mathcal{P}_{j}\right\}_{j=0,1,2, \ldots}$ of refining dyadic partitions (thus the atoms of $\mathcal{P}_{j}$ are $d$-intervals of size $\sim 2^{-j}$ ) and denote $E_{j}$ the corresponding expectation operators. Denote also $\tau_{\theta}$ the shift operators on $\mathbb{T}^{d}$.

We perform the following construction. Given $F \in H^{s}\left(\Omega ; S^{1}\right)$, denote $F_{\theta}=F \circ \tau_{\theta}$ and $\varphi[\theta]$ the lifting of $F_{\theta}$ gotten from the construction described in Section 1 (with fixed $\mathcal{P}_{j}$ 's). Thus

$$
F_{\theta}=e^{i \varphi[\theta]} \text { and } F=e^{i\left(\varphi[\theta] \circ \tau_{-\theta}\right)}
$$

and $\varphi[\theta] \circ \tau_{-\theta}=\varphi$ is a lifting for $F$. Thus Theorem 4 will follow immediately from the next statement. 
Lemma E.1. We have

$$
\int_{\mathbb{T}^{d}}\|\varphi[\theta]\|_{H^{s}} d \theta \leq C(1-2 s)^{-1 / 2}\|F\|_{H^{s}}
$$

Proof. We show in fact that

$$
\int\|\varphi[\theta]\|_{H^{s}}^{2} d \theta \leq C(1-2 s)^{-1}\|F\|_{H^{s}}^{2}
$$

The lefthand side of (E.4) equals

$$
\begin{aligned}
& \iiint \frac{\left|\varphi[\theta]-\tau_{h} \varphi[\theta]\right|^{2}(x)}{|h|^{2 s+d}} d x d h d \theta \\
\sim & \sum_{j \geq 0} 2^{(2 s+d) j} \iint_{|h| \sim 2^{-j}}\left\|\varphi[\theta]-\tau_{h} \varphi[\theta]\right\|_{2}^{2} d h d \theta .
\end{aligned}
$$

Denote $\varphi[\theta]$ by $\varphi$ for simplicity. Fix $j$.

Writing

$$
\varphi=E_{j} \varphi+\sum_{j^{\prime}>j} \Delta_{j^{\prime}} \varphi \quad\left(\Delta_{j^{\prime}}=E_{j^{\prime}}-E_{j^{\prime}-1}\right)
$$

estimate

$$
\left\|\varphi-\tau_{h} \varphi\right\|_{2}^{2} \lesssim\left\|E_{j} \varphi-\tau_{h} E_{j} \varphi\right\|_{2}^{2}+\sum_{j^{\prime}>j}\left(j^{\prime}-j\right)^{2}\left\|\Delta_{j^{\prime}} \varphi\right\|_{2}^{2}
$$

Recall inequality (1.5) in Section 1

$$
\left|\varphi_{j}-\varphi_{j-1}\right| \leq C\left(\left|F_{\theta}-E_{j}\left(F_{\theta}\right)\right|+\left|F_{\theta}-E_{j-1}\left(F_{\theta}\right)\right|\right) .
$$

Hence, since $\varphi_{j}=E_{j}\left(\varphi_{j}\right)$, we have

$$
\begin{aligned}
\left\|\Delta_{j} \varphi\right\|_{2} & \leq\left\|E_{j}\left(\varphi-\varphi_{j}\right)\right\|_{2}+\left\|E_{j-1}\left(\varphi-\varphi_{j-1}\right)\right\|_{2}+\left\|\varphi_{j}-\varphi_{j-1}\right\|_{2} \\
& \leq C \sum_{j^{\prime} \geq j}\left\|\varphi_{j^{\prime}}-\varphi_{j^{\prime}-1}\right\|_{2} \\
& \leq C \sum_{j^{\prime} \geq j-1}\left\|F_{\theta}-E_{j^{\prime}}\left(F_{\theta}\right)\right\|_{2} \\
& \leq C \sum_{j^{\prime} \geq j-1}\left(j^{\prime}-j+2\right)\left\|\Delta_{j^{\prime}} F_{\theta}\right\|_{2}
\end{aligned}
$$


and estimate in (E.7)

$$
\left\|\Delta_{j^{\prime}} \varphi\right\|_{2}^{2} \leq C \sum_{j^{\prime \prime} \geq j^{\prime}-1}\left(j^{\prime \prime}-j^{\prime}+2\right)^{4}\left\|\Delta_{j^{\prime \prime}} F_{\theta}\right\|_{2}^{2}
$$

Thus the contribution of the second term in (E.7) is bounded by

$$
\begin{aligned}
& \sum_{j \geq 0} 2^{(2 s+d) j} \iint_{|h| \sim 2^{-j}}\left\{\sum_{j^{\prime}>j}\left(j^{\prime}-j\right)^{2}\left\|\Delta_{j^{\prime}} \varphi\right\|_{2}^{2}\right\} d h d \theta \\
& \leq C \int d \theta\left\{\sum_{j \geq 0} 2^{2 s j} \sum_{j^{\prime \prime}+2 \geq j^{\prime}>j}\left(j^{\prime}-j\right)^{2}\left(j^{\prime \prime}-j^{\prime}+2\right)^{4}\left\|\Delta_{j^{\prime \prime}} F_{\theta}\right\|_{2}^{2}\right\} \\
& \leq C \int d \theta\left\{\sum_{j^{\prime \prime}>0} 2^{2 s j^{\prime \prime}}\left\|\Delta_{j^{\prime \prime}} F_{\theta}\right\|_{2}^{2}\right\} .
\end{aligned}
$$

Recalling the proof of Theorem A1 (in particular the inequality $Y \leq C X$ independent of the assumption $2 s<1$ ) we have

$$
(E .12) \leq C \int d \theta\left\|F_{\theta}\right\|_{H^{s}}^{2} \leq C\|F\|_{H^{s}}^{2}
$$

Thus the $\theta$-integration is irrelevant here.

The main point is the contribution of the first term $\left\|E_{j} \varphi-\tau_{h} E_{j} \varphi\right\|_{2}^{2}$ in (E.5), thus

$$
\sum_{j \geq 0} 2^{(2 s+d) j} \iint_{|h| \sim 2^{-j}} \int\left|E_{j} \varphi-\tau_{h} E_{j} \varphi\right|^{2} d \theta d h d x .
$$

Estimate

$$
\left|E_{j} \varphi-\tau_{h} E_{j} \varphi\right| \leq \sum_{j^{\prime} \leq j}\left|\Delta_{j^{\prime}} \varphi-\tau_{h} \Delta_{j^{\prime}} \varphi\right|
$$

Write

$$
\Delta_{j^{\prime}} \varphi=\sum_{I \in \mathcal{P}_{j^{\prime}}} a_{I} \chi_{I}
$$

Then, for $|h|<2^{-j}$, one easily verifies that

$$
\left|\Delta_{j^{\prime}} \varphi-\tau_{h} \Delta_{j^{\prime}} \varphi\right| \leq \sum_{I \in \mathcal{P}_{j^{\prime}}}\left|a_{I}\right|\left|\chi_{I}-\tau_{h} \chi_{I}\right| \leq C\left(\left|\Delta_{j^{\prime}} \varphi\right| * P_{2^{-j^{\prime}}}\right) \chi_{j^{\prime}, 2^{-j}}
$$


where $\chi_{j^{\prime}, 2^{-j}}$ denotes the characteristic function of the set

$$
\left\{x ; \operatorname{dist}(x, \partial I) \leq 2^{-j} \text { for some } I \in \mathcal{P}_{j^{\prime}}\right\}
$$

and $P_{\varepsilon}$ denotes the usual Poisson-kernel for instance.

Thus

$$
\int \chi_{j^{\prime}, 2^{-j}}=\operatorname{mes}(E .18) \leq C 2^{j^{\prime} d} 2^{-j^{\prime}(d-1)} 2^{-j} \leq C 2^{j^{\prime}-j}
$$

Substituting (E.17) in (E.15) implies (since $\cup_{I \in \mathcal{P}_{j_{1}^{\prime}}} \partial I \subset \cup_{I \in \mathcal{P}_{j_{2}^{\prime}}} \partial I$ for $j_{1}^{\prime}<j_{2}^{\prime}$ )

$$
\left|E_{j} \varphi-\tau_{h} E_{j} \varphi\right|^{2} \leq \sum_{\substack{j_{1}^{\prime} \leq j, j_{2}^{\prime} \leq j \\ j_{1}^{\prime} \leq j_{2}^{\prime}}}\left(\left|\Delta_{j_{1}^{\prime}} \varphi\right| * P_{2^{-j_{1}^{\prime}}}\right)\left(\left|\Delta_{j_{2}^{\prime}} \varphi\right| * P_{2^{-j_{2}^{\prime}}}\right) \chi_{j_{1}^{\prime}, 2^{-j}}
$$

Next,

$$
\Delta_{j^{\prime}} \varphi=E_{j^{\prime}}\left(\varphi-\varphi_{j^{\prime}}\right)-E_{j^{\prime}-1}\left(\varphi-\varphi_{j^{\prime}-1}\right)+\varphi_{j^{\prime}}-\varphi_{j^{\prime}-1}
$$

and again from inequality (E.8)

$$
\left|\varphi-\varphi_{j^{\prime}}\right| \leq C \sum_{j^{\prime \prime}>j^{\prime}}\left(j^{\prime \prime}-j^{\prime}\right)\left|\Delta_{j^{\prime \prime}} F_{\theta}\right|
$$

We get

$$
\left|\Delta_{j^{\prime}} \varphi\right| * P_{2^{-j^{\prime}}} \leq C \sum_{j^{\prime \prime} \geq j^{\prime}}\left(j^{\prime \prime}-j^{\prime}+1\right)\left(\left|\Delta_{j^{\prime \prime}} F_{\theta}\right| * P_{2^{-j^{\prime}}}\right)
$$

Substituting (E.23) in (E.20) and then in (E.14) gives

$\sum_{j \geq 0} 2^{2 s j} \iint d x d \theta \sum_{\substack{j_{1}^{\prime} \leq j, j_{2}^{\prime} \leq j, j_{1}^{\prime} \leq j_{2}^{\prime} \\ j_{1}^{\prime \prime} \geq j_{1}^{\prime}, j_{2}^{\prime \prime} \geq j_{2}^{\prime}}}\left(j_{1}^{\prime \prime}-j_{1}^{\prime}+1\right)\left(j_{2}^{\prime \prime}-j_{2}^{\prime}+1\right)\left(\left|\Delta_{j_{1}^{\prime \prime}} F_{\theta}\right| * P_{2^{-j_{1}^{\prime}}}\right)\left(\left|\Delta_{j_{2}^{\prime \prime}} F_{\theta}\right| * P_{2^{-j_{2}^{\prime}}}\right) \chi_{j_{1}^{\prime}, 2^{-j}}(x)$.

The role of the $\theta$-translation is that we introduced an extra variable to estimate (E.24). Write $F$ as a Fourier series in $\mathbb{T}^{d}$

$$
F=\sum_{n \in \mathbb{Z}^{d}} \widehat{F}(n) e^{i n x}
$$

Then

$$
\begin{aligned}
\Delta_{j}\left(F_{\theta}\right) & =\sum \widehat{F}(n) e^{i n \theta} \Delta_{j}\left(e^{i n \cdot}\right) \\
\left|\left(\left|\Delta_{j} F_{\theta}\right| * P_{\varepsilon}\right)(x)\right|^{2} & \leq \int\left|\sum \widehat{F}(n) e^{i n \theta} \Delta_{j}\left(e^{i n \cdot}\right)(x-y)\right|^{2} P_{\varepsilon}(y) d y .
\end{aligned}
$$


Integrating (E.26) in $\theta$ gives clearly

$$
\left\|\left|\Delta_{j} F_{\theta}\right| * P_{\varepsilon}\right\|_{L_{\theta}^{2}}^{2} \leq \sum|\widehat{F}(n)|^{2}\left\|\Delta_{j}\left(e^{i n .}\right)\right\|_{\infty}^{2} \lesssim \sum|\widehat{F}(n)|^{2}\left(1 \wedge|n| 2^{-j}\right)^{2} .
$$

To estimate (E.24), perform first the $\theta$-integration using Cauchy-Schwarz and (E.27).

This gives, recalling (E.19)

$$
\begin{aligned}
\sum_{j \geq 0} 2^{2 s j} \sum_{j_{\alpha}^{\prime} \leq j, j_{\alpha}^{\prime} \leq j_{\alpha}^{\prime \prime}, j_{1}^{\prime} \leq j_{2}^{\prime}} 2^{j_{1}^{\prime}-j}\left(j_{1}^{\prime \prime}-j_{1}^{\prime}+1\right)\left(j_{2}^{\prime \prime}-j_{2}^{\prime}+1\right)\left[\sum_{n}|\widehat{F}(n)|^{2}\left(1 \wedge|n| 2^{-j_{1}^{\prime \prime}}\right)^{2}\right]^{1 / 2} & {\left[\sum_{n}|\widehat{F}(n)|^{2}\left(1 \wedge|n| 2^{-j_{2}^{\prime \prime}}\right)^{2}\right]^{1 / 2} . }
\end{aligned}
$$

To evaluate (E.28), denote

$$
\begin{aligned}
& \ell_{\alpha}=j_{\alpha}^{\prime \prime}-j_{\alpha}^{\prime} \geq 0 \quad(\alpha=1,2) \\
& m=j_{2}^{\prime}-j_{1}^{\prime} \geq 0
\end{aligned}
$$

so that

$$
\begin{aligned}
(E .28)= & \sum_{m, \ell_{1} \cdot \ell_{2} \geq 0}\left(\ell_{1}+1\right)\left(\ell_{2}+1\right) \sum_{j_{1}^{\prime}} 2^{j_{1}^{\prime}}\left(\sum_{j \geq j_{1}^{\prime}} 2^{(2 s-1) j}\right) . \\
& {\left[\sum_{n}|\hat{F}(n)|^{2}\left(1 \wedge|n| 2^{-j_{1}^{\prime}-\ell_{1}}\right)^{2}\right]^{1 / 2}\left[\sum_{n}|\hat{F}(n)|^{2}\left(1 \wedge|n| 2^{-j_{1}^{\prime}-m-\ell_{2}}\right)^{2}\right]^{1 / 2} . }
\end{aligned}
$$

Applying Cauchy-Schwarz for the $j_{1}^{\prime}$-summation

$$
\begin{aligned}
(E .31) & \leq C \sum_{m, \ell_{1}, \ell_{2}}\left(\ell_{1}+1\right)\left(\ell_{2}+1\right)(1-2 s)^{-1} \\
& {\left[\sum_{n, j_{1}^{\prime}}|\hat{F}(n)|^{2} 2^{2 s j_{1}^{\prime}}\left(1 \wedge|n| 2^{-j_{1}^{\prime}-\ell_{1}}\right)^{2}\right]^{1 / 2}\left[\sum_{n, j_{1}^{\prime}} \mid \hat{F}(n)^{2} 2^{2 s j_{1}^{\prime}}\left(1 \wedge|n| 2^{-j_{1}^{\prime}-m-\ell_{2}}\right)^{2}\right]^{1 / 2} . }
\end{aligned}
$$

Writing

$$
\sum_{j} 2^{2 s j}\left(1 \wedge|n| 2^{-j-\ell}\right)^{2} \sim 2^{-2 s \ell}(1+|n|)^{2 s}
$$

it follows that

$$
\begin{aligned}
(E .32) & \leq \frac{C}{1-2 s} \sum_{m, \ell_{1}, \ell_{2}}\left(\ell_{1}+1\right)\left(\ell_{2}+1\right) 2^{-s\left(\ell_{1}+\ell_{2}+m\right)}\left(\sum_{n}|\hat{F}(n)|^{2}(1+|n|)^{2 s}\right) \\
& \leq C(1-2 s)^{-1}\|F\|_{H^{s}}^{2} .
\end{aligned}
$$


Since (E.5) is bounded by the sum of (E.13) and (E.34), this proves Lemma E.1.

Remark E.1. The optimality of the bound (E.2) when $d=2$ was proved in Remark 7 . The case $d \geq 3$ is similar by choosing

$$
g(x)=\frac{\left(x_{1}, x_{2}\right)}{\left(x_{1}^{2}+x_{2}^{2}\right)^{1 / 2}} \quad x=\left(x_{1}, x_{2}, \ldots, x_{d}\right)
$$

and proceeding as in the 2-dimensional case. The optimality of (E.2) when $d=1$ is more delicate and will be established in the forthcoming paper Bourgain, Brezis and Mironescu $[1]$.

Remark E.2. Theorem E.1 is still valid if the cube $Q$ is replaced by a smooth domain $\Omega$ in $\mathbb{R}^{d}, d \geq 2$ (without any topological assumption on $\Omega$ ). The proof can be modified as follows. Consider a neighborhood $\tilde{\Omega}$ of $\bar{\Omega}$ and a function still denoted $F, F \in H^{s}\left(\tilde{\Omega} ; S^{1}\right)$ which extends the original $F$ (this can be done by the standard procedure of local reflexion across the boundary). Next, construct a finite sequence of disjoint cubes $\left(Q_{\alpha}\right)$, having the same size, and such that $\Omega \subset \bigcup_{\alpha} Q_{\alpha} \Subset \tilde{\Omega}$. The construction described in Section 1 is still valid on $\bigcup_{\alpha} Q_{\alpha}$ and we obtain a lifting $\varphi \in H^{s}\left(\bigcup_{\alpha} Q_{\alpha} ; \mathbb{R}\right)$. For $\theta \in \mathbb{R}^{d}$ with $|\theta|<\delta, \delta$ sufficiently small, $F_{\theta}=F \circ \tau_{\theta}$ is well defined on $\bigcup_{\alpha} Q_{\alpha}$ has a lifting $\varphi[\theta]$. The proof of Lemma E.1 described above can be adapted and yields

$$
\int_{\mid \theta \|<\delta}\|\varphi[\theta]\|_{H^{s}} d \theta \leq C(1-2 s)^{-1 / 2}\|F\|_{H^{s}}
$$

Theorem E.1 is also valid if the cube $Q$ is replaced by a smooth $d$-dimensional manifold $M, d \geq 1$, say without boundary. The dyadic partition of $Q$ is replaced by some dyadic "triangulation" of $M$. The shift operators $\tau_{\theta}$ are replaced by a finite family $\left\{S_{i}(t)\right\}$, $1 \leq i \leq N$ of 1-parameter group of transformations on $M$ such that, at each $x \in M$, the generators $V_{i}(x)=\frac{d}{d t} S_{i}(t) x_{\left.\right|_{t=0}}$ span the tangent space $T_{x}(M)$. Such a family can be easily constructed as integral curves for the differential equations $\dot{x}(t)=V_{i}(x(t))$ and the vectorfields $V_{i}(x)$ are obtained via local coordinates and a partition of unity. The shift operators $\tau_{\theta}$ are replaced by the shifts along the $S_{i}$, i.e., $\sigma_{\theta}=\prod_{i} S_{i}\left(t_{i}\right)$, where $\theta=\left(t_{1}, t_{2}, \ldots, t_{N}\right)$, and then $F_{\theta}=F \circ \sigma_{\theta}$. Adapting the proof of Lemma E.1 we find

$$
\int_{\theta \in \mathbb{R}^{N},|\theta|<1}\|\varphi[\theta]\| d \theta \leq C(1-2 s)^{-1 / 2}\|F\|_{H^{s}}
$$

\section{APPENDIX F}

\section{Martingale representation and lifting in $\mathbf{H}^{\mathbf{s}, \mathbf{p}}$}


The question of representation and lifting can be raised in other function spaces. For instance, in the $H^{s, p}$ space.

Recall the definition of the $H^{s, p}$-norm $(0<s<1)$

$$
\|f\|_{H^{s, p}}=\left[\int\left(\int \frac{|f(x+h)-f(x)|^{2}}{|h|^{2 s+d}} d h\right)^{p / 2} d x\right]^{1 / p} .
$$

This space is a bit more delicate to deal with then $W^{s, p}$. The natural martingale counterpart of (F.1) is given by

$$
\left\|\left(\sum 2^{2 j s}\left|\Delta_{j} f\right|^{2}\right)^{1 / 2}\right\|_{p}
$$

where $\Delta_{j} f=E_{j}(f)-E_{j-1}(f)$ and $E_{j}$ is the conditional expectation operator with respect to $\mathcal{P}_{j}$ (as before). This situation is a bit different from $W^{s, p}$. We show the following

Proposition F.1. (i) We have

$$
\left\|\left(\sum 4^{j s}\left|\Delta_{j} f\right|^{2}\right)^{1 / 2}\right\|_{p} \leq C \mid f \|_{H^{s, p}}
$$

(ii) If $s p<1$ and $p \geq 2$, then the converse inequality holds

$$
\|f\|_{H^{s, p}} \leq C\left\|\left(\sum 4^{j s}\left|\Delta_{j} f\right|^{2}\right)^{1 / 2}\right\|_{p}
$$

(iii) Inequality (F.4) fails for $s>\frac{1}{2}$.

Proposition F.1 leaves some cases unanswered and they will possibly be addressed elsewhere. Again, Proposition F.1 is relevant to the question of Triebel [1] concerning the representation of Besov and Sobolev spaces in the Haar-system. It implies that for the spaces $H^{s, p}=F_{p, 2}^{s}$, the conjecture is valid if $p s<1, p \geq 2$ but fails for $s>\frac{1}{2}$.

In the proof of Proposition F.1, we will make use of some standard martingale inequalities (which the reader may find in Garsia [1] for instance). 
Proposition F.2. We have

$$
\left\|\sum E_{j}\left(g_{j}\right)\right\|_{p} \leq C_{p}\left\|\sum\left|g_{j}\right|\right\|_{p} \text { for } 1 \leq p<\infty
$$

and

$$
\left\|\left(\sum\left|E_{j}\left(g_{j}\right)\right|^{2}\right)^{1 / 2}\right\|_{p} \leq C_{p}\left\|\left(\sum\left|g_{j}\right|^{2}\right)^{1 / 2}\right\|_{p} \text { for } 1<p<\infty
$$

In both statements, the sequence $\left\{g_{j}\right\}$ consists of arbitrary functions.

Remark F.1. In (F.5), (F.6), the expectation operators $E_{j}$ may get replaced by convolution operator $P_{2^{-j}}$ for instance, where $P_{\varepsilon}$ stands for the usual Poisson kernel (cf. Stein [1]).

Proof of Proposition F.1.

(i) By (F.6)

$$
\left\|\left(\sum 4^{j s}\left|\Delta_{j} f\right|^{2}\right)^{1 / 2}\right\|_{p} \leq C\left\|\left(\sum 4^{j s}\left|f-E_{j-1}(f)\right|^{2}\right)^{1 / 2}\right\|_{p}
$$

Again

$$
\begin{aligned}
\left|\left(f-E_{j-1}(f)\right)(x)\right| & \leq 2^{j d} \int_{|h|<2^{-j}}|f(x)-f(x+h)| d h \\
\left|f-E_{j-1}(f)\right|^{2} & \leq 2^{j d} \int_{|h|<2^{-j}}\left|f-\tau_{h} f\right|^{2} d h .
\end{aligned}
$$

where $\tau_{h}$ is the translation operator.

Substituting (F.8) in (F.7) implies

$$
\begin{aligned}
(F .7) & \leq\left\|\left\{\int d h\left|f-\tau_{h} f\right|^{2}\left[\sum_{|h|<2^{-j}} 4^{j s} 2^{j d}\right]\right\}^{1 / 2}\right\|_{p} \\
& \sim\left\|\left\{\int\left|f-\tau_{h} f\right|^{2}|h|^{-(d+2 s)} d h\right\}^{1 / 2}\right\| \|_{p} \\
& =\|f\|_{H^{s, p}} .
\end{aligned}
$$


(ii) Write

$$
\int\left|f-\tau_{h} f\right|^{2}|h|^{-(d+2 s)} d h \sim \sum_{j} 2^{j(d+2 s)} \int_{|h| \sim 2^{-j}}\left|f-\tau_{h} f\right|^{2} d h .
$$

Fix $j$. Estimate

$$
\left|f-\tau_{h} f\right| \leq\left|f_{j}-\tau_{h} f_{j}\right|+\left|f-f_{j}\right|+\tau_{h}\left|f-f_{j}\right|
$$

$$
\left|f-\tau_{h} f\right|^{2} \lesssim \sum_{j^{\prime}<j}\left(j-j^{\prime}\right)^{2}\left|\Delta_{j^{\prime}} f-\tau_{h}\left(\Delta_{j^{\prime}} f\right)\right|^{2}+\left|f-f_{j}\right|^{2}+\tau_{h}\left|f-f_{j}\right|^{2}
$$

and substituting (F.11) in (F.10), we get the following contributions

$$
\begin{aligned}
(F .10) & \leq C \sum_{j^{\prime}<j} 2^{j(d+2 s)}\left(j-j^{\prime}\right)^{2} \int_{|h| \sim 2^{-j}}\left|\Delta_{j^{\prime}} f-\tau_{h}\left(\Delta_{j^{\prime}} f\right)\right|^{2} d h \\
& +\sum_{j} 4^{j s}\left|f-f_{j}\right|^{2} \\
& +\sum_{j} 4^{j s}\left[P_{2^{-j}} *\left(\left|f-f_{j}\right|^{2}\right)\right] .
\end{aligned}
$$

\section{Contribution of (F.13)}

Write

$$
\begin{aligned}
\left\|(F .13)^{1 / 2}\right\|_{p} & \leq\left\|\left[\sum_{j} 4^{j s} \sum_{j^{\prime} \geq j}\left(j^{\prime}-j\right)^{2}\left|\Delta_{j^{\prime}} f\right|^{2}\right]^{1 / 2}\right\|_{p} \\
& \sim\left\|\left(\sum_{j} 4^{j^{\prime} s}\left|\Delta_{j^{\prime}} f\right|^{2}\right)^{1 / 2}\right\|_{p}
\end{aligned}
$$

\section{Contribution of (F.14)}

$$
\left\|(F .14)^{1 / 2}\right\|_{p}=\left\{\int\left\{\sum_{j} 4^{j s}\left[P_{2^{-j}} *\left(\left|f-f_{j}\right|^{2}\right)\right]\right\}^{p / 2}\right\}^{\frac{1}{p}} .
$$

Use the general inequality (see Remark F.1)

$$
\left\|\sum_{j} P_{2^{-j}} g_{j}\right\|_{q} \leq C_{q}\left\|\sum_{j}\left|g_{j}\right|\right\|_{q} \text { for } 1 \leq q<\infty
$$


Thus, since $p \geq 2$, letting $q=p / 2$ in (F.17), it follows

$$
\begin{aligned}
(F .16) & \leq C\left[\int\left(\sum_{j} 4^{j s}\left|f-f_{j}\right|^{2}\right)^{p / 2}\right]^{1 / p} \\
& \leq C\left\|\left(\sum_{j} 4^{j s}\left|\Delta_{j} f\right|^{2}\right)^{1 / 2}\right\|_{p}
\end{aligned}
$$

\section{Contribution of (F.12)}

Denoting $\ell=j-j^{\prime} \geq 0$, write

$$
\left\|(F .12)^{1 / 2}\right\|_{p} \leq \sum_{\ell \geq 0} \ell 2^{\ell s}\left(\left\|\left[\sum_{j^{\prime}} 4^{j^{\prime} s}\left(2^{\left(j^{\prime}+\ell\right) d} \int_{|h| \leq 2^{-\left(j^{\prime}+\ell\right)}}\left|\Delta_{j^{\prime}} f-\tau_{h}\left(\Delta_{j^{\prime}} f\right)\right|^{2} d h\right)\right]^{1 / 2}\right\|_{p} .\right.
$$

To bound (F.19), fix $\ell$ and consider the map

$$
T_{\ell}: L_{\ell^{2}}^{p} \rightarrow L_{L_{h}^{2} \ell^{2}}^{p}
$$

defined by

$$
T_{\ell} \bar{g}=T_{\ell}\left(\left\{g_{j}\right\}\right)=\left\{\left(E_{j} g_{j}-\tau_{h} E_{j} g_{j}\right) 2^{(j+\ell) d / 2} \chi_{\left[|h|<2^{-(j+\ell)}\right]}\right\}
$$

Thus the components of $T_{\ell} \bar{g}$ are functions of $x$ and $h$.

Denote $\left\|T_{\ell}\right\|_{p}$ the norm of (F.20). We estimate $\left\|T_{\ell}\right\|_{p}, 2 \leq p$, by interpolation between 2 and some large $q$.

Fixing $2<q<\infty$, we may bound

$$
\begin{aligned}
\left\|T_{\ell} \bar{g}\right\|_{L_{L_{h}^{2} \ell^{2}}} & \leq\left\|E_{j}\left|g_{j}\right| .2^{(j+\ell) d / 2} \chi_{\left[|h|<2^{-(j+\ell)]}\right.}\right\|_{L_{L_{h}^{q} \ell^{2}}}+\left\|\tau_{h}\left(E_{j}\left|g_{j}\right|\right) .2^{(j+\ell) d / 2} \chi_{\left[|h|<2^{-(j+\ell)]}\right.}\right\|_{L_{L_{h}^{q} \ell^{2}}} \\
& =(F .22)+(F .23) .
\end{aligned}
$$

Thus, invoking (F.6)

$$
(F .22) \sim\left\|\left[\sum\left(E_{j}\left|g_{j}\right|\right)^{2}\right]^{1 / 2}\right\|_{q} \leq C_{q}\|\bar{g}\|_{L_{\ell^{2}}^{q}}
$$


Also, since $q>2$ and using inequalities (F.17), (F.6)

$$
\begin{aligned}
(F .23) & \leq C\left\|\left[\sum_{j}\left(E_{j}\left|g_{j}\right|\right)^{2} * P_{2^{-(j+\ell)}}\right]^{1 / 2}\right\|_{q}=\left\|\sum_{j}\left(E_{j}\left|g_{j}\right|\right)^{2} * P_{2^{-(j+\ell)}}\right\|_{q / 2}^{1 / 2} \\
& \leq C\left\|\sum_{j}\left(E_{j}\left|g_{j}\right|\right)^{2}\right\|_{q / 2}^{1 / 2} \leq C\|\bar{g}\|_{L_{\ell^{2}}^{q}}
\end{aligned}
$$

Thus $\left\|T_{\ell} \bar{g}\right\|_{L_{L_{h}^{2} \ell^{2}}} \leq C_{q}\|\bar{g}\|_{L_{\ell^{2}}^{q}}$, i.e.

$$
\left\|T_{\ell}\right\|_{q} \leq C_{q} \text { for } 2 \leq q<\infty
$$

Next, for $p=2$, a direct calculation gives

$$
\begin{aligned}
\left\|T_{\ell} \bar{g}\right\|_{L_{x}^{2} L_{h}^{2} \ell^{2}} & =\left[\sum_{j} 2^{(j+\ell) d} \iint_{|h|<2^{-(j+\ell)}}\left|\left(E_{j} g_{j}\right)(x)-\left(E_{j} g_{j}\right)(x+h)\right|^{2} d x d h\right]^{1 / 2} \\
& \leq C 2^{-\ell / 2}\left(\sum_{j}\left\|E_{j} g_{j}\right\|_{2}^{2}\right)^{1 / 2} \\
& \leq C 2^{-\ell / 2}\|\bar{g}\|_{L_{\ell^{2}}^{2}}
\end{aligned}
$$

The estimate (F.28) simply results from the fact that for $I \in \mathcal{P}_{j}$ and $|h|<2^{-(j+\ell)}$

$$
\left\|\chi_{I}(x)-\chi_{I}(x+h)\right\|_{L_{x}^{2}} \leq C 2^{(-d-1) j / 2-\frac{j+\ell}{2}}=C 2^{-\ell / 2} 2^{-d j / 2} .
$$

¿From (F.29),

$$
\left\|T_{\ell}\right\|_{2} \leq C 2^{-\ell / 2}
$$

Interpolating $2<p<q$, it results from (F.26), (F.31) that

$$
\left\|T_{\ell}\right\|_{p}<C_{\varepsilon} 2^{-\ell\left(\frac{1}{p}-\varepsilon\right)} \text { for all } \varepsilon>0 .
$$

Returning to (F.19), we define thus

$$
g_{j^{\prime}}=2^{j^{\prime} s} \Delta_{j^{\prime}} f
$$


so that, by (F.32)

$$
\begin{aligned}
(F .19) & \leq \sum_{\ell \geq 0} \ell 2^{\ell s}\left\|T_{\ell}\left\{g_{j^{\prime}}\right\}\right\|_{L_{L_{h}^{2} \ell^{2}}^{p}} \\
& \leq C_{\varepsilon} \sum_{\ell \geq 0} \ell 2^{\ell s} 2^{-\ell\left(\frac{1}{p}-\varepsilon\right)}\left\|\left\{g_{j^{\prime}}\right\}\right\|_{L_{\ell^{2}}^{p}} .
\end{aligned}
$$

Since $s p<1$, we may take $\varepsilon$ sufficiently small to ensure boundedness of the factor in (F.34), leading again to the bound $\left\|\left(\sum 4^{j^{\prime} s}\left|\Delta_{j} f\right|^{2}\right)^{1 / 2}\right\|_{p}$.

This establishes inequality (F.4).

(iii) Take $d=1$ and define

$$
f_{j}=2^{-j s} \sum_{r=1}^{2^{j}}(-1)^{r} \chi_{I_{r}} \text { where } \mathcal{P}_{j}=\left\{I_{1}, \ldots, I_{2^{j}}\right\} .
$$

Fix a large integer $R$ and let $\left\{j_{r}\right\}_{r=1, \ldots, R}$ be a lacunary sequence.

Define

$$
f=\sum_{r=1}^{R} \varepsilon_{r} f_{j_{r}}
$$

where $\varepsilon_{r}= \pm 1$ are independent signs. Thus $\Delta_{j_{r}} f=\varepsilon_{r} f_{j_{r}}$ and trivially

$$
\left\|\left(\sum 4^{j s}\left|\Delta_{j} f\right|^{2}\right)^{1 / 2}\right\|_{p}=R^{1 / 2}
$$

Next, take $\delta>0$ a small number and write

$$
\int\left|f-\tau_{h} f\right|^{2}|h|^{-(1+2 s)} d h \geq \sum_{r=1}^{R}\left(\delta 2^{-j_{r}}\right)^{-(1+2 s)} \int_{|h|<\delta 2^{-j_{r}}}\left|f-\tau_{h} f\right|^{2} d h .
$$

Averaging over the $\pm \operatorname{signs} \varepsilon_{r}$ in (F.36) permits us clearly to ensure that

$$
(F .38) \geq \sum_{r}\left(\delta 2^{-j_{r}}\right)^{-(1+2 s)} \int_{|h|<\delta 2^{-j_{r}}}\left|f_{j_{r}}-\tau_{h} f_{j_{r}}\right|^{2} d h .
$$


Recalling (F.35), one sees that

$$
\begin{aligned}
(F .39) & \geq c \sum_{r}\left(\delta 2^{-j_{r}}\right)^{-(1+2 s)}\left(\delta 2^{-j_{r}}\right) 4^{-j_{r} s} \sum_{I \in \mathcal{P}_{j_{r}}} \chi_{\left[\operatorname{dist}(x, \partial I)<\frac{1}{2} \delta 2^{-j_{r}}\right]} \\
& =c \delta^{-2 s} \sum_{r} \sum_{I \in \mathcal{P}_{j_{r}}} \chi_{\left[\operatorname{dist}(x, \partial I)<\frac{1}{2} \delta|I|\right]}
\end{aligned}
$$

Therefore

$$
\|f\|_{H^{s, p}} \geq c \delta^{-s}\left\|\left\{\sum_{r=1}^{R} \sum_{I \in \mathcal{P}_{j_{r}}} \chi_{\left[\operatorname{dist}(x, \partial I)<\frac{1}{2} \delta|I|\right]}\right\}^{1 / 2}\right\|_{p}
$$

Fixing $\delta>0$ and letting $R>R(\delta)$ be sufficiently large, the reader will easily convince himself that

$$
(F .42) \geq c \delta^{-s}(\delta R)^{1 / 2}=c \delta^{\frac{1}{2}-s} \cdot(F .37)
$$

Consequently, letting $\delta \rightarrow 0$, we see that inequality (F.4) cannot hold for $s>\frac{1}{2}$. This completes the proof of Proposition F.1.

There is the following application of Proposition F.1 to the lifting problem of unimodular functions.

Corollary F.1. Let $s>0, s p<1, p \geq 2$ and $F \in H^{s, p}\left(\Omega ; S^{1}\right)$, where $\Omega$ is a cube in $\mathbb{R}^{d}$. Then

$$
F=e^{i \varphi}
$$

for some $\varphi \in H^{s, p}(\Omega)$.

Remark F.2. The other cases not covered by the corollary have not been investigated.

Proof. The function $\varphi$ is constructed as in the $W^{s, p}$-case (see Section 1). ¿From Proposition F.1, $(i),(i i)$ and similar calculations as in the $W^{s, p}$-estimate, we obtain (with the 
notations from Section 1)

$$
\begin{aligned}
\|\varphi\|_{H^{s, p}} & \leq C\left\|\left(\sum 4^{j s}\left|\Delta_{j} \varphi\right|^{2}\right)^{1 / 2}\right\|_{p} \\
& \leq C\left\|\left(\left.\sum 4^{j s} E_{j}\left(\varphi-\varphi_{j}\right)\right|^{2}\right)^{1 / 2}\right\|_{p}+\left\|\left(\sum 4^{j s}\left|\varphi_{j}-\varphi_{j-1}\right|^{2}\right)^{1 / 2}\right\|_{p} \\
& \quad \text { by }{ }^{(F .6)} C\left\|\left(\sum 4^{j s}\left|\varphi-\varphi_{j}\right|^{2}\right)^{1 / 2}\right\|_{p}+\left\|\left(\sum 4^{j s}\left|\varphi_{j}-\varphi_{j-1}\right|^{2}\right)^{1 / 2}\right\|_{p} \\
& \leq C\left\|\left(\sum_{j^{\prime}>j} 4^{j s}\left(j^{\prime}-j\right)^{2}\left|\varphi_{j^{\prime}}-\varphi_{j^{\prime}-1}\right|^{2}\right)^{1 / 2}\right\|_{p} \\
& \mathrm{~b} y(1.5) \\
& \leq C\left\|\left(\sum_{j^{\prime}>j} 4^{j s}\left(j^{\prime}-j\right)^{2}\left|F-E_{j^{\prime}-1} F\right|^{2}\right)^{1 / 2}\right\| \\
& \leq C\left\|\left(\sum_{j^{\prime \prime} \geq j^{\prime}>j} 4^{j s}\left(j^{\prime}-j\right)^{2}\left(j^{\prime \prime}-j^{\prime}+1\right)^{2}\left|\Delta_{j^{\prime \prime}} F\right|^{2}\right)^{1 / 2}\right\|_{p} \\
& \leq C\left\|\left(\sum_{j^{\prime \prime}} 4^{j^{\prime \prime} s}\left|\Delta_{j^{\prime \prime}} F\right|^{2}\right)^{1 / 2}\right\| \\
& \leq C\|F\|_{H^{s, p} .}
\end{aligned}
$$

Acknowledgment: The second author (H.B.) is partially supported by a European Grant ERB FMRX CT98 0201. He is also a member of the Institut Universitaire de France. We thank Y. Meyer and G. Bourdaud for providing us useful references.

\section{REFERENCES}

[[XXXX] R. Adams [1], Sobolev Spaces, Acad. Press, 1975.

S. Alinhac and P. Gérard [1], Opérateurs pseudo-différentiels et théorème de Nash-Moser, Interéditions, 1991.

F. Bethuel [1], The approximation problem for Sobolev maps between two manifolds, Acta Math. 167 (1991), 153-206.

F. Bethuel, H. Brezis and F. Hélein [1], Ginzburg-Landau Vortices, Birkhäuser, Boston and Basel, 1997. 
F. Bethuel and F. Demengel [1], Extensions for Sobolev mappings between manifolds, Cal. Var. PDE 3 (1995), 475-491.

F. Bethuel and X. Zheng [1], Density of smooth functions between two manifolds in Sobolev spaces, J. Funct. Anal. 80 (1988), 60-75.

J.-M.Bony [1], Calcul symbolique et propagation des singularités pour les équations aux dérivées partielles nonlinéaires, Ann. Sc. Ec. Norm Sup. 14 (1981), 209-246.

G. Bourdaud [1], Ondelettes et espaces de Besov, Revista Mat. Iberoamericana 11 (1995), 477-511.

J. Bourgain, H. Brezis and P. Mironescu [1], in preparation.

A. Boutet de Monvel-Berthier, V. Georgescu and R. Purice [1], Sur un problème aux limites de la théorie de Ginzburg-Landau, C.R. Acad. Sc. Paris 307 (1988), 55-58; [2], A boundary value problem related to the Ginzburg-Landau model, Comm. Math, Phys, 142 (1991), 1-23.

H, Brezis [1], Analyse fonctionnelle: théorie et applications, Masson, 1983.

H. Brezis, Y. Li, P. Mironescu and L. Nirenberg [1], Degree and Sobolev spaces, Top. Methods in Nonlinear Analysis (to appear).

H. Brezis and L. Nirenberg [1], Degree Theory and BMO, Part I: Compact manifolds without boundaries, Selecta Math. 1 (1995), 197-263; [2] Part II: Compact manifolds with boundaries, Selecta Math. 2 (1996), 309-368.

G. Carbou [1], Applications harmoniques à valeurs dans un cercle, C. R. Acad. Sc., Paris 1992, 359-362.

J.-Y. Chemin [1], Fluides parfaits incompressibles, vol. 230, Astérisque, 1995.

R. Coifman and Y. Meyer [1], Une généralisation du théorème de Calderón sur l'intégrale de Cauchy, in Fourier Analysis Proc. Sem. at El Escorial, Asoc. Mat. Española, Madrid, 1980, 88-116.

R. Devore and V. A. Popov [1], Interpolation of Besov spaces, Trans. Amer. Math. Soc. 305 (1988), 397-414.

M. Escobedo [1], Some remarks on the density of regular mappings in Sobolev classes of $S^{M}$-valued functions, Rev. Mat. Univ. Complutense, Madrid 2 (1988), 127-144.

J. Garnett, and P. Jones [1], BMO from dyadic BMO, Pacific J. Math 99 (1982), 351-371.

A. Garsia [1], Martingale Inequalities: Seminar notes on recent progress, Benjamin, 1973.

R. Hardt, D. Kinderlehrer and F. H. Lin [1], The variety of configurations of static liquid crystals, in Variational Methods (H. Berestycki, J.-M. Coron and I. Ekeland, eds.), Birkhäuser, 1990.

F.-H. Lin and T. Rivière [1], Complex Ginzburg-Landau Equations in high dimensions and codimension two area minimizing currents, J. Eur. Math. Soc. 1 (1999), 237-311, Erratum, to appear. 
J.L. Lions and E. Magenes [1], Problèmes aux limites non homogènes, Dunod, 1968; English translation, Springer, 1972.

V. Maz’ja [1], Sobolev Spaces, Springer, 1985.

L. Nirenberg [1], On elliptic partial differential equations, Ann. Sc. Norm. Pisa 13 (1959), 115-162.

T. Rivière [1], Lignes de tourbillons dans le modèle abélien de Higgs, CRAS 321 (1995), 73-76; [2], Line vortices in the U(1)- Higgs model, COCV 1 (1996), 77-167; [3], Dense subsets of $H^{1 / 2}\left(S^{2} ; S^{1}\right)$, Global analysis and Geometry (to appear).

E. Sandier [1], Ginzburg-Landau minimizers from $\mathbb{R}^{n+1}$ to $\mathbb{R}^{n}$ and minimal connections, preprint (1999).

T. Runst [1], Mapping properties of non-linear operators in spaces of Triebel-Lizorkin and Besov type, Analysis Mathematica 12 (1986), 313-346.

E. Stein [1], Topics in Harmonic Analysis Related to the Littlewood-Paley Theory, Princeton Univ. Press, 1970.

H. Triebel [1], Spline bases and spline representations in function spaces, Arch. Math. 36 (1981), 348-359; [2] Theory of function spaces, Birkhäuser, 1983.

(1) Institute for Advanced Study

PRINCETON, NJ 08540

E-mail address: : bourgain@ias.edu

(2) Analyse Numérique

Université P. et M. Curie, B.C. 187

4 PL. Jussieu

75252 Paris Cedex 05

E-mail address: brezis@ccr.jussieu.fr

(3) Rutgers University

Dept. of Math., Hill Center, Busch Campus

110 Frelinghuysen Rd, Piscataway, NJ 08854

E-mail address: brezis@math.rutgers.edu

(4) Departement de Mathématiques

UnIVERSITÉ PARIS-SUd

91405 ORSAY

E-mail address: Petru.Mironescu@math.u-psud.fr 